\title{
CURVE CROSSING IN A PROTEIN: COUPLING OF THE ELEMEN- TARY QUANTUM PROCESS TO MOTIONS OF THE PROTEIN
}

\author{
KLAUS SCHULTEN \\ Department of Physics and Beckman Institute \\ University of Illinois at Urbana-Champaign \\ Urbana, IL 61801 \\ USA
}

\section{Introduction}

The quintessential quantum process in biology and chemistry involves electrons switching between two states. Two examples are electron transfer reactions in proteins when an electron moves from an orbital on the donor moiety $\mathrm{D}$ to an orbital on the acceptor moiety $\mathrm{A}$ and bond formation or bond breaking in an enzyme when electrons shift from a non-bonding state to a bonding state or vice versa. The energy expectation values of the two states $E_{1}(t)$ and $E_{2}(t)$ vary in time due to motions along a reaction coordinate, but also due to thermal fluctuations of the remaining degrees of freedom of the combined reaction-protein system. Often the interaction energies which couple the two electronic states involved in the reaction are weak, i.e., are small compared to the temporal variations of $E_{1}(t)$ and $E_{2}(t)$. In this rather typical case the actual reaction process is confined to moments when the two electronic states become energetically degenerate $\left[E_{1}(t)=E_{2}(t)\right]$ or, to use a widely accepted phrase, when the curves $E_{1}$ and $E_{2}$ cross.

In this lecture I will discuss curve crossing specifically in a protein environment. We will study how interactions between a two-dimensional quantum system and a protein matrix affect the curve crossing process. In particular, we will demonstrate how the influence of the protein on the process can be captured succinctly through stochastic quantum mechanics. Such description allows one to separate protein dynamics and the evolution of the quantum system: a molecular dynamics simulation is carried out to characterize the motion of the protein; the results of the simulations are then employed in a stochastic quantum mechanical calculation involving only the quantum mechanical degrees of freedom. The best known and most widely studied curve crossing process, electron transfer, will serve as an example.

The major part of this lecture has been reported in $[1,2,3]$. Section 5 is taken from notes of my Theoretical Biophysics Course given during 1982-1987 at the Technical University of Munich.

Three advantages arise from the suggested description. First, and most obvi-

ous, the procedure is computationally and conceptually simpler than combining a quantum mechanical and a molecular dynamics description into a single calculation. 
Second, and equally important, such description focusses on the essential contribution of the protein environment to the quantum system and, thereby, yields a better understanding of the reaction studied. Lastly, one can consider the molecular dynamics simulations, carried out classically, as the high temperature limit of a quantum mechanical description and use the results for a fully quantum mechanical description in which the protein matrix is represented as a bath of quantum mechanical oscillators, suitably chosen to match the results of molecular dynamics simulations.

The description provided here does not account for forces which the quantum system exerts back onto the classical system. This deficiency is insignificant in those cases in which many degress of freedom of the protein matrix are weakly coupled to the quantum process. This situation arises, for example, in electron transfer, in which case long-range Coulomb forces couple essentially the whole protein matrix to the quantum process; in this case the effect of the protein motion on the electron transfer involves many small, additive contributions such that a back-reaction of the quantum system becomes actually immaterial. However, in case that some degrees of freedom of the protein matrix are strongly affected by the quantum system such that the curve crossing event induces forces which correlate the protein motion with the evolution of the quantum system, one must resort to a description combining quantum mechanics and classical molecular dynamics as outlined in other sections of these proceedings. But even if such behaviour arises, which is expected to be the case only for a small number of degrees of freedom, one is left with a need to account for contributions of the remaining degrees of freedom, for which purpose one may want to resort to a description as outlined below.

The lecture describes first the case in which the force generated by the quantum system and influencing the protein is not neglected. Representing the protein as an ensemble of harmonic oscillators permits one to solve the response of the protein. This renders a closed evolution equation for the density matrix of the quantum system which exhibits a non-linear term accounting for the effect of the quantum system onto the protein and back onto the quantum system. The latter term is neglected in the following sections and two approaches are considered. In a first approach the protein matrix is represented as a classical system which contributes time-dependent secular terms to the Hamiltonian of the quantum system [1]. We demonstrate how these terms affect the curve crossing process, resorting to the well-known Kubo theory of line shapes [4]. We show how the stochastic process which represents the time dependence of the stated secular terms can be characterized through an independent molecular dynamics simulation. We focus, in particular, on the case that the stochastic process is of the Ornstein-Uhlenbeck type, i.e., corresponds to a Gaussian equilibrium distribution of diagonal energies; in this case a simple analytical description for the quantum system can be given. In a second approach [2, 3], often referred to as the spin-boson model, we consider the case that the protein matrix is represented through a bath of quantum mechanical oscillators linearly coupled to the quantum system, but not subject to energy redistribution. We show that the overall effect of 
such bath depends on average quantities which, at physiological temperatures, can be rather readily obtained from classical molecular dynamics simulations. The second approach can be shown to become equivalent to the first approach in certain limits, e.g., at high (physiological) temperatures and for line shape functions which are wide compared to the frequencies involved in energy redistribution among protein vibrational modes, limits which prevail in case of electron transfer in proteins. We complete the lecture with a discussion of applications of the suggested descriptions.

\section{The Generic Model: Two-State Quantum Sys- tem Coupled to an Oscillator}

The model we consider first is extremely simple, yet it encapsulates the essence of a reaction process in a protein governed by a quantum mechanical curve crossing process. The reaction process connects a state $|1\rangle$ and a state $|2\rangle$, e.g., two electronic states of a substrate. Examples are (i) an electron transfer process from a donor D to an acceptor $\mathrm{A}$, where $|1\rangle$ and $|2\rangle$ represent the states before electron transfer, i.e., $\mathrm{A} \mathrm{D}$, and after electron transfer, i.e., $\mathrm{A}^{-} \mathrm{D}^{+}$, and (ii) bond breaking in which case $|1\rangle$ describes the electronic state corresponding to a covalent bond and $|2\rangle$ describes the electronic state corresponding to the broken bond.

In the following we adopt the representation

$$
|1\rangle=\left(\begin{array}{l}
1 \\
0
\end{array}\right), \quad|2\rangle=\left(\begin{array}{l}
0 \\
1
\end{array}\right)
$$

such that the Hamiltonian of the quantum system is

$$
\hat{H}=\left(\begin{array}{cc}
E_{1} & v \\
v & E_{2}
\end{array}\right)
$$

where $v$ denotes the coupling between the states $|1\rangle$ and $|2\rangle$ and where $E_{1,2}$ are the associated energy expectation values of these states. The essential aspect of the quantum system is that it is coupled to the protein matrix through a dependence of

the elements of $\hat{H}$ on the state of the protein matrix. For the sake of simplicity we assume here that only the energies $E_{1,2}$ depend on the protein environment and that $v$ is constant. Also, we represent presently the protein through a single harmonic oscillator. This oscillator exhibits its coupling to the quantum system through a shift of its equilibrium position in going from state $|1\rangle$ to state $|2\rangle$. Such behaviour is captured by the energy functions

$$
\begin{aligned}
& E_{1}=\frac{p^{2}}{2 m}+\frac{1}{2} m \omega^{2} q^{2} \\
& E_{2}=\frac{p^{2}}{2 m}+\frac{1}{2} m \omega^{2}\left(q-q_{o}\right)^{2}
\end{aligned}
$$


Replacing the diagonal elements of the Hamiltonian in (2) by these expressions evidently leads to a dependence of the quantum system on the momentum and position of the Hamiltonian representing the protein environment.

Following the procedure for quantum/classical molecular dynamics simulations described in [5] and elsewhere in these proceedings one can state the equation of motion for both the density matrix $\varrho(t)$ of the two state quantum system and of the oscillator. It holds

$$
\begin{aligned}
\dot{\varrho} & =\frac{i}{\hbar}[\varrho, \hat{H}] \\
m \ddot{q} & =-\operatorname{tr}\left(\varrho \frac{\partial}{\partial q} \hat{H}\right)
\end{aligned}
$$

where (5) is the Pauli equation and (6) the Newtonian equation of motion where the force of the classical particle is evaluated according to the Hellman-Feynman theorem; $\operatorname{tr} A$ denotes the trace of the operator $\mathrm{A}$. In the following we wish to describe the situation that the quantum system is initially, i.e., at $t=0$, in state $|1\rangle$ such that the Pauli equation (5) is solved subject to the initial condition

$$
\varrho(0)=\left(\begin{array}{ll}
1 & 0 \\
0 & 0
\end{array}\right)
$$

This initial condition implies, in particular,

$$
[\varrho(t)]_{22}=0, \quad t \leq 0 .
$$

Evaluation of the force exerted onto the oscillator, i.e., the r.h.s. of (6), yields

$$
-\operatorname{tr}\left(\varrho \frac{\partial}{\partial q} \hat{H}\right)=-m \omega^{2} q+\varrho_{22}(t) c
$$

where we introduced the expression

$$
q_{o}=c / m \omega^{2}
$$

and used $\varrho_{11}(t)+\varrho_{22}(t)=1$. Inserting this into (6) leads to

$$
\ddot{q}+\omega^{2} q=\frac{c}{m} \varrho_{22}(t) .
$$

Equation (11) describes a forced oscillator. Defining

$$
\xi=\dot{q}+i \omega q
$$

and

$$
F(t)=\frac{c}{m} \varrho_{22}(t)
$$


one can write (11) as a first order differential equation

$$
\dot{\xi}-i \omega \xi=F(t) \text {. }
$$

We note that, according to $(8)$, holds $F(t)=0, t \leq 0$.

We want to solve now Eqs. (12-14) in case that the harmonic oscillator, at $t \leq 0$, has not been coupled yet to the quantum system and exhibits a time-dependence

$$
q(t)=A_{o} \sin (\omega t+\delta), \quad t \leq 0
$$

The corresponding form of $\xi(t)$, defined in (12), is

$$
\xi_{o}(t)=\xi(0) e^{i \omega t}, \quad t \leq 0, \quad \xi(0)=\omega A_{0} e^{i \delta}
$$

The solution of (14) which matches this functional behaviour is for $t \geq 0$

$$
\begin{aligned}
\xi(t) & =\xi_{o}(t)+\delta \xi(t) . \\
\delta \xi(t) & =\int_{0}^{t} d t^{\prime} \frac{c}{m} \varrho_{22}\left(t^{\prime}\right) e^{i \omega\left(t-t^{\prime}\right)}
\end{aligned}
$$

where $\delta \xi(t)$ describes the effect of the force (13) on the oscillator. This expression can be inserted into the Pauli equation (5). Noting

$$
\begin{aligned}
& E_{1}=\frac{1}{2} m|\xi(t)|^{2} \\
& E_{2}=\frac{1}{2} m\left|\xi(t)-i \omega q_{o}\right|^{2}
\end{aligned}
$$

we obtain the non-linear evolution equation

$\dot{\varrho}=\frac{i}{\hbar}\left[\varrho,\left(\begin{array}{cc}\frac{1}{2} m\left|\xi_{o}(t)+\int_{0}^{t} d t^{\prime} \frac{c}{m} \varrho_{22}\left(t^{\prime}\right) e^{i \omega\left(t-t^{\prime}\right)}\right|^{2} & v \\ v & \frac{1}{2} m\left|\xi_{o}(t)+\int_{0}^{t} d t^{\prime} \frac{c}{m} \varrho_{22}\left(t^{\prime}\right) e^{i \omega\left(t-t^{\prime}\right)}-i \omega q_{o}\right|^{2}\end{array}\right)\right]$.

The evolution equation (21) accounts for the effect of the quantum system on the protein (oscillator) and its back-reaction onto the quantum system. The treatment of this system has been discussed in the lecture by Berendsen in this conference. The description provided can be generalized to a situation in which the protein is represented by an ensemble of harmonic oscillators. In this case the interaction between the quantum process and the protein matrix spreads over many degrees of freedom. For some of these degrees of freedom the coupling might be strong such that it cannot be neglected. For most degrees of freedom the coupling should be so weak that it's effect, in particular, in the concert of the overall motion of the protein, can be neglected. In the following sections we will disregard the perturbation $\delta \xi(t)$ 
[cf. (18)] on the quantum system and replace the non-linear evolution equation (21) by the linear evolution equation

$$
\dot{\varrho}=\frac{i}{\hbar}\left[\varrho,\left(\begin{array}{cc}
\frac{1}{2} m\left|\xi_{o}(t)\right|^{2} & v \\
v & \frac{1}{2} m\left|\xi_{o}(t)-i \omega q_{o}\right|^{2}
\end{array}\right)\right] .
$$

This equation accounts for an effect of the harmonic oscillator on the quantum system. The simplification introduced will allow us to generalize our treatment in several ways. We can replace the single oscillator by an ensemble of oscillators, in fact, even by an ensemble of quantum mechanical oscillators. We can also represent the ensemble of classical oscillators by a random process governing the time-dependent matrix elements in the Hamiltonian in (22). We will demonstrate further that the essential characteristics of the ensemble of oscillators representing the protein marix can be obtained from classical molecular dynamics simulations. These generalizations and the connection to molecular dynamics simulations are a most welcome feature of the theory presented below. Nevertheless, it appears desirable to include in these generalizations the back-reaction of the quantum system on the environmental dynamics as described by (21).

\section{Two-State System Coupled to a Classical Medium $[1]$}

In this section we assume a two-state quantum system with energies (secular terms) $E_{1}$ and $E_{2}$ which depend on the conformation of the whole protein described through the vector $\mathbf{R}(t) \in \mathbb{R}^{3 N}$ for the case of a protein with $N$ atoms. The Hamiltonian reads then

$$
\tilde{H}_{I}=\left(\begin{array}{cc}
E_{1}[\mathbf{R}(t)] & v \\
v & E_{2}[\mathbf{R}(t)]
\end{array}\right)
$$

We assume that the protein motion captured by $\mathbf{R}(t)$ leads to curve crossing events, i.e., to situations $t=t^{\prime}$ in which $E_{1}\left[\mathbf{R}\left(t^{\prime}\right)\right]=E_{2}\left[\mathbf{R}\left(t^{\prime}\right)\right]$ holds. The matrix element $v$ induces transitions between the two states of the quantum system. We assume in the following that $v$ is time-independent, an assumption made for the sake of simplicity, but which ought to be relaxed in a more complete theory. We want to assume also that the system has a finite life time $\tau_{o}$ in state $|2\rangle$. This property will be accounted for by an operator

$$
K_{I}=\left(\begin{array}{cc}
0 & 0 \\
0 & \tau_{o}^{-1}
\end{array}\right)
$$

which, save for a factor $\hbar$, represents an optical potential.

The goal of the theory is to determine the $2 \times 2$ density matrix $\tilde{\varrho}(t)$ which obeys

$$
\partial_{t} \tilde{\varrho}(t)=\frac{i}{\hbar}\left[\tilde{\varrho}(t), \tilde{H}_{I}(t)\right]_{-}-\left[\tilde{\varrho}(t), K_{I}\right]_{+} .
$$


where [, ]- presents the commutator and [, $]_{+}$presents the anti-commutator. For an evaluation of $\tilde{\varrho}(t)$ it is helpful to adopt a new basis. Let $(\alpha(t) \beta(t))^{T}$ denote the state of the quantum system at time $t$. One introduces the new basis through the scalar transformation

$$
\left(\begin{array}{l}
\alpha^{\prime}(t) \\
\beta^{\prime}(t)
\end{array}\right)=u_{o}(t)\left(\begin{array}{c}
\alpha(t) \\
\beta(t)
\end{array}\right)
$$

where $u_{o}(t)$ is a scalar function which obeys the differential equation

$$
\partial_{t} u_{o}(t)=\frac{i}{\hbar} E_{1}[\mathbf{R}(t)] u_{o}(t), \quad u_{o}(0)=1
$$

One can demonstrate that the density matrix in the new representation obeys a Pauli equation with Hamiltonian

$$
H_{I}=\left(\begin{array}{cc}
0 & v \\
v & \Delta E[\mathbf{R}(t)]
\end{array}\right)
$$

and

$$
\Delta E[\mathbf{R}(t)]=E_{2}[\mathbf{R}(t)]-E_{1}[\mathbf{R}(t)]
$$

leaving (24) unmodified. The new representation yields the same values for the diagonal elements of the new density matrix $\varrho(t)$ as the density matrix $\tilde{\varrho}(t)$ in the old representation, i.e., the probabilities to find the system in state $|1\rangle$ or state $|2\rangle$ are identical in the old and new representation.

The density matrix $\varrho(t)$ obeys the Liouville equation

$$
\partial_{t} \varrho(t)=\frac{i}{\hbar}\left[H_{I}(t), \varrho(t)\right]_{-}-[\mathcal{K}, \varrho(t)]_{+} .
$$

This equation shows that a description of the coupling between the protein matrix and the two-state quantum system can be reduced to an evaluation of the so-called energy gap function $\epsilon(t)=\Delta E[\mathbf{R}(t)]$ from a molecular dynamics simulation.

To link a molecular dynamics simulation to the quantum system, one monitors in the molecular dynamics simulation the function $\epsilon(t)$ at time instances $t=t_{1}, t_{2}, \ldots$ and employs the resulting values for an evaluation of $\varrho(t)$. Such evaluation is based on a solution of Eq. (30) for a piecewise time-independent Hamiltonian $\tilde{H}_{I}$

$$
\varrho(t+\Delta t)=\mathcal{P} \varrho(t) \mathcal{P}^{\dagger}, \mathcal{P}=\exp \left[\Delta t\left(\frac{i}{\hbar} \tilde{H}_{I}-\tilde{K}_{I}\right)\right]
$$

Evaluating the exponential operator $\mathcal{P}$ through Taylor expansion and grouping terms of odd and even powers yields [1]

$$
\varrho(t+\Delta t)=\left(\cos \Delta t \gamma \mathbf{1}+i \frac{\sin \Delta t \gamma}{\gamma} \mathbf{A}\right) \varrho(t)\left(\cos \Delta t \bar{\gamma} \mathbf{1}-i \frac{\sin \Delta t \bar{\gamma}}{\bar{\gamma}} \overline{\mathbf{A}}\right) e^{-\Delta t / \tau_{o}}
$$


where 1 stands for the identity matrix, $\bar{\gamma}, \bar{A}$ denote the complex conjugate of $\gamma, A$, and the abbreviations

$$
\gamma=\sqrt{\Omega^{2}+\omega^{2}}, \omega=V / \hbar, \Omega=\frac{1}{2 \hbar}\left(\Delta E_{M D}-\frac{i \hbar}{\tau_{o}}\right), \mathbf{A}=\left(\begin{array}{cc}
\Omega & \omega \\
\omega & -\Omega
\end{array}\right)
$$

are used. Corresponding calculations have been carried out in [1]. The fluctuations of the energy gap $\epsilon(t)$ as a controlling factor for the rate of electron transfer in the photosynthetic reaction center had been studied by means of molecular dynamics simulations as soon as the structure of this protein had been discovered $[6,7]$. A further molecular dynamics analysis in terms of the Marcus theory of electron transfer $[8,9]$ (see also below) has been provided in [10].

In case of electron transfer reactions in proteins the interaction matrix element $v$ in $(1,23,28)$ accounts for the electronic tunneling between electron donor and electron acceptor moieties. The evaluation of $v$ for electron tunneling pathways in proteins has been studied extensively by Onuchic as described elsewhere in these proceedings.

To evaluate the density matrix $\varrho(t)$ one carries out a classical molecular dynamics simulation which provides one with the vector $\mathbf{R}(t)$ describing the momentaneous protein conformation. One determines then the corresponding energy gap $\Delta E[\mathbf{R}(t)]$ for which purpose one obviously needs to know the expressions for the energies $E_{1}[\mathbf{R}(t)]$ and $E_{2}[\mathbf{R}(t)]$. For example, in case of an electron transfer reaction state $|1\rangle$ describes an electron configuration $\mathrm{AD}$ and state $|2\rangle$ an electron configuration $\mathrm{A}^{-} \mathrm{D}^{+}$ where $\mathrm{A}$ and $\mathrm{D}$ are electron acceptor and donor moieties in the protein. Knowing the atomic partial charges corresponding to $\mathrm{AD}$ and to $\mathrm{A}^{-} \mathrm{D}^{+}$one can evaluate $E_{1}[\mathbf{R}(t)]$ and $E_{2}[\mathbf{R}(t)]$ as the Coulomb energies of the acceptor and donor moieties with the protein matrix, to which one adds the redox energies of the states $\mathrm{AD}$ and $\mathrm{A}^{-} \mathrm{D}^{+}$. Unfortunately, a particular molecular dynamics simulation leading to a particular history of $\mathbf{R}(t)$-values is not necessarily representative and one needs to average either over the dynamics started with many different initial conditions (ensemble average) or carry out a suitable time average.

The situation, which we wish to describe, starts from the quantum system in state $|1\rangle$, i.e., from [c.f. (7)]

$$
\varrho(0)=\left(\begin{array}{ll}
1 & 0 \\
0 & 0
\end{array}\right)
$$

The system will transfer to state $|2\rangle$ in which state it decays with life time $\tau_{o} / 2$ such that, ultimately, state $|1\rangle$ will be completely depleated. In case that $\tau$ is sufficiently short and $\Delta E[\mathbf{R}(t)]$ is random one expects that the population of state $|1\rangle$ exhibits an approximate exponential decay

$$
\varrho_{11}(t) \approx \exp \left(-k_{\mathrm{cl}} t\right) .
$$

The decay constant $k_{\mathrm{cl}}$ may be evaluated through

$$
\left[k_{\mathrm{cl}}\right]^{-1} \approx \int_{0}^{\infty} d t \varrho_{11}(t) .
$$




\section{Two State System Coupled to a Stochastic Medium [1]}

A difficulty of the description outlined in Sect. 3 is the need to average the density matrix $\varrho(t)$ over many histories of the energy gap function $\Delta E[\mathbf{R}(t)]$. In this section we link the molecular dynamics simulations to the quantum system through an interpretation of $\Delta E[\mathbf{R}(t)]$ as a stochastic process. In this case one can describe the evolution of the quantum system to second order in $v$, as defined in $(23,28)$, averaged over all possible histories of the protein. Beside being eminently useful in applications the description provided in this section makes it also transparent which characteristics of the fluctuations of $\Delta E[\mathbf{R}(t)]$ are essential for the control of the quantum system by the protein.

We consider here the simplest case that the properties of $\epsilon(t)=\Delta E[\mathbf{R}(t)]$ can be captured by a so-called Ornstein-Uhlenbeck process $[11,12]$. This stochastic process is characterized through three properties. The first characteristic property is the average value

$$
\langle\epsilon\rangle=\frac{1}{N_{t}} \sum_{j=1}^{N_{t}} \epsilon\left(t_{j}\right) .
$$

The second characteristic property implies that the distribution of values $\epsilon\left(t_{1}\right), \epsilon\left(t_{2}\right) \ldots$ is Gaussian such that the distribution is completely characterized through the root mean square deviation $\sigma$ defined through

$$
\sigma^{2}=\frac{1}{N_{t}} \sum_{j=1}^{N_{t}} \epsilon^{2}\left(t_{j}\right)-\langle\epsilon\rangle^{2} .
$$

The third characteristic property is related to the normalized correlation function of the energy gap

$$
C_{\epsilon \epsilon}(t)=\frac{1}{\sigma^{2}}\left(\langle\epsilon(t) \epsilon(0)\rangle-\langle\epsilon\rangle^{2}\right) .
$$

This function is conventionally evaluated through

$$
C_{\epsilon \epsilon}(t)=\frac{1}{\sigma^{2}} \frac{1}{M} \sum_{\alpha=1}^{M}\left(\left\langle\epsilon\left(t+t_{\alpha}\right) \epsilon\left(t_{\alpha}\right)\right\rangle-\langle\epsilon\rangle^{2}\right)
$$

for, e.g., $M=100$, where $t_{j}$ denotes time instances along the simulations which are spaced far apart, e.g., 10 ps. Obviously, $C_{\epsilon \epsilon}(t)$ captures the dynamic properties of $\epsilon(t)$. The present description in terms of an Ornstein-Uhlenbeck process assumes that $C_{\epsilon \epsilon}(t)$ is well described by a single exponential decay, i.e., by

$$
C_{\epsilon \epsilon}(t) \approx \exp (-t / \tau)
$$

such that the dynamics is presented sufficiently through a single decay time $\tau$. 
A quantity which conforms to the three characteristics of the Ornstein-Uhlenbeck process is distributed according to a probablity distribution $p(\epsilon, t)$ which obeys the Fokker-Planck equation

$$
\partial_{t} p(\epsilon, t)=\mathcal{L} p(\epsilon, t), \mathcal{L}=\frac{\sigma^{2}}{2 \tau} \partial_{\epsilon} p_{0}(\epsilon) \partial_{\epsilon}\left[p_{0}(\epsilon)\right]^{-1}
$$

where

$$
p_{o}(\epsilon)=\frac{1}{\sqrt{2 \pi \sigma}} \exp \left[-(\epsilon-\langle\epsilon\rangle)^{2} / 2 \sigma^{2}\right] .
$$

The assumption that $\epsilon(t)$, as monitored in a simulation, obeys an Ornstein-Uhlenbeck process is made here mainly for the sake of simplicity. The general framework of the theory presented allows other stochastic processes to represent the coupling of the protein matrix to the quantum system. We note that the Ornstein-Uhlenbeck process actually describes a Brownian harmonic oscillator [12].

By employing the theory in $[13,14]$ one can express the density matrix

$$
\varrho(t)=\int d \Delta E^{\prime} \mathcal{I}\left(\Delta E^{\prime}\right) \varrho_{o}\left(\Delta E^{\prime} \mid t\right)
$$

where

$$
\varrho_{o}\left(\Delta E^{\prime} \mid t\right)=\exp \left[-\frac{i t}{\hbar}\left(\begin{array}{cc}
0 & v \\
v & \Delta E^{\prime}-i \hbar / \tau
\end{array}\right)\right]\left(\begin{array}{ll}
1 & 0 \\
0 & 0
\end{array}\right) \exp \left[\frac{i t}{\hbar}\left(\begin{array}{cc}
0 & v \\
v & \Delta E^{\prime}+i \hbar / \tau
\end{array}\right)\right]
$$

Here $\varrho_{o}\left(\Delta E^{\prime} \mid t\right)$ represents the density matrix for a Hamiltonian (25) with a timeindependent energy term $\Delta E^{\prime}$, i.e., it can be evaluated by means of $(32,33)$, applying these formulas for arbitrary $d t . \mathcal{I}\left(\Delta E^{\prime}\right)$ is the Kubo line shape function [4]

$$
\mathcal{I}\left(\Delta E^{\prime}\right)=\frac{1}{\pi} \operatorname{Re}\left\langle 0\left|\frac{1}{\frac{i}{\hbar}\left(u-\Delta E^{\prime}\right)-\mathcal{L}}\right| 0\right\rangle .
$$

Expressions (44-46) yield an approximation, accurate to third order in $v$, for the diagonal elements of $\varrho(t)$. However, the approximation includes all orders of $v$ in order to conserve the trace of the density matrix and to ascertain convergence to the exact $\varrho(t)$ in the limits of slow or fast stochastic motion measured on a time scale $\hbar / v[4]$. A method for the evaluation of the line shape function (46) is described in the appendix. Other computational strategies are decribed in $[15,13,16,17,18]$. A systematic approximation to matrix elements of the inverse of Fokker-Planck operators is provided by the generalized moment expansion. This approach is described systematically in [19] where it is considered, however, for real operators; a generalization to complex operators, as they occur in (46), is straightforward.

The aim of the present theory is to determine the time-dependence of the occupancy of state $|1\rangle$ which is initially populated, i.e., to determine $\left[\varrho\left(\Delta E^{\prime} \mid t\right)\right]_{11}$. As in 
the description in Sect. 3 this state eventually should become completely unpopulated and we assume a behaviour described by a mono-exponential decay

$$
\left.\left[\varrho\left(\Delta E^{\prime} \mid t\right)\right]_{11} \approx \exp \left[-k_{c l}^{S T}\left(\Delta E^{\prime}\right) t\right)\right] .
$$

We assume the same approximation for the $(1,1)$ element of $\varrho_{o}\left(\Delta E^{\prime} \mid t\right)$, i.e.,

$$
\left[\varrho_{o}\left(\Delta E^{\prime} \mid t\right)\right]_{11} \approx \exp \left[-k_{o}\left(\Delta E^{\prime}\right) t\right]
$$

and can establish then $k_{o}\left(\Delta E^{\prime}\right)$ through

$$
\left[k_{o}\left(\Delta E^{\prime}\right)\right]^{-1} \approx \int_{0}^{\infty} d t\left[\varrho_{o}\left(\Delta E^{\prime} \mid t\right)\right]_{11}
$$

which applies if (48) holds exactly. Note that we do not define the rates through the time derivative of the density matrix element itself since, of course, this derivative vanishes at $t=0$. One can conclude then from Eq. (44)

$$
\exp \left[-k_{c l}^{S T} t\right] \approx \int d \Delta E^{\prime} \mathcal{I}\left(\Delta E^{\prime}\right) \exp \left[-k_{o}\left(\Delta E^{\prime}\right) t\right]
$$

Differentiating both sides at $t=0$ yields

$$
k_{c l}^{S T} \approx \int d \Delta E^{\prime} \mathcal{I}\left(\Delta E^{\prime}\right) k_{o}\left(\Delta E^{\prime}\right)
$$

In the present case three parameters determine the operator $\mathcal{L}$ in $(46,42)$ and, hence, the functional form of $\mathcal{I}\left(\Delta E^{\prime}\right)$, namely, $\langle\epsilon\rangle, \sigma^{2}$, and $\tau$ defined in (37), (38) and in $(41,42)$, respectively. $\langle\epsilon\rangle$ determines the center of $\mathcal{I}\left(\Delta E^{\prime}\right)$ on the $\Delta E^{\prime}$ axis. The parameter $\sigma^{2}$ can be absorbed into the scale of the $\Delta E^{\prime}$-axis. The corresponding line shape function is then, using $u^{\prime}=u / \sigma, \Delta \epsilon=\Delta E^{\prime} / \sigma$,

$$
\mathcal{I}(\Delta \epsilon) \sim \operatorname{Re}\left\langle 0\left|\frac{1}{i\left(u^{\prime}-\Delta \epsilon\right)-\rho \partial_{u^{\prime}} p_{o}\left(u^{\prime}\right) \partial_{u^{\prime}}\left[p_{o}\left(u^{\prime}\right)\right]^{-1}}\right| 0\right\rangle
$$

which expresses the line shape function in dimensionless variables. Here,

$$
\rho=\frac{\hbar}{2 \tau \sigma}
$$

is the single dimensionless parameter which describes the dynamic effect of fluctuations in $\Delta E(t)$, large $\rho$ values corresponding to rapid fluctuations. Since the effect of $\langle\epsilon>$ and $\sigma$ can be absorbed through shift and scaling of the $\Delta E$-axis, the parameter $\rho$ constitutes the only parameter which determines the shape of $\mathcal{I}(\Delta E)$ and, hence, the effect of the protein matrix on the quantum system. In case of the photosynthetic reaction center of Rhodopseudomonas viridis studied in [1] a value $\rho=0.03$ was determined which is so small that a motional narrowing of the distribution $\mathcal{I}\left(\Delta E^{\prime}\right)$ relative to the wide equilibrium distribution of $\Delta E$ can be neglected, i.e., $\mathcal{I}\left(\Delta E^{\prime}\right)$ is described well assuming that the protein matrix is characterized solely through an equilibrium distribution of all of its degrees of freedom. We will study a respective model for the protein matrix in the next two sections. However, in case of a narrow equilibrium distribution of $\Delta E$, i.e., with a width of the order of $\hbar / \tau$ or smaller, one would expect that the fluctuations of $\Delta E(t)$ will modify $\mathcal{I}(\Delta E)$ significantly. 


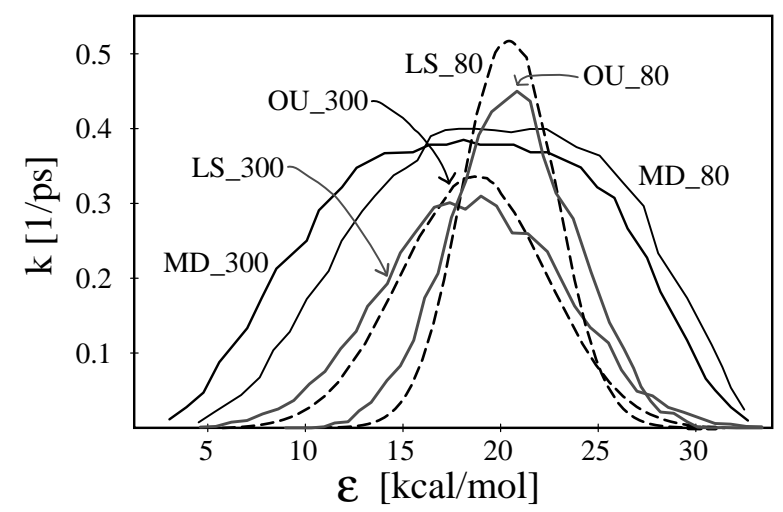

Figure 1: Electron transfer rates $k_{c l}(\epsilon)$ evaluated by means of molecular dynamics simulation and the theory in Sect. 3 at 300 and at $80 \mathrm{~K}$. In the calculations the redox energy $E=\epsilon$ was varied as shown on the horizontal axis. The corresponding rate constants are labelled MD_300 and MD_80. The figure shows also rate constants evaluated according to the theory in Sect. 3, except that the data for the energy gap $\Delta E$ were generated by means of an Ornstein-Uhlenbeck process employing random numbers. The corresponding rates are labelled OU_300 and OU_80. A third set of rate constants $k_{c l}^{S T}$, labelled ST_300 and ST_80, has been calculated according to the theory in Sect. 4, i.e., according to Eq. (51) (dashed lines). From [1].

Applications The theory outlined in this and in the previous Section has been applied in the framework of molecular dynamics studies of the photosynthetic reaction center of Rh. viridis [14, 1]. Figure 1 compares the resulting electron transfer rates. The theory in the present Section has also been applied to describe the dynamics of radical pairs connected through a polymer chain which mediates a time-dependent exchange interaction $[15,13]$. In the latter case the polymer folding motions, analyzed through molecular dynamics simulations, induces a stochastic variation of the exchange interaction. The theory has been applied also to magnetic resonance imaging microscopy $[17,18,20]$. In this case the spins of water protons, diffusing in a magnetic field gradient, experience a stochastic separation of their spin-up and spindown states. This leads to an NMR spectrum which depends on the diffusion space and on the Brownian motion of water; the spectrum, in a proper analysis, reveals the geometry of the diffusion space. In $[15,13,17,18]$ the Kubo line shape function had been evaluated numerically through a discretization of the operator $\mathcal{L}$ in (46). The algorithm presented there, e.g., in [18], can be applied to a class of stochastic processes governed by a so-called Smoluchowski equation. These processes cover Gaussian and non-Gaussian equilibrium distributions $p_{o}(\epsilon)$. 


\section{Two State System Coupled to a Single Quantum Mechanical Oscillator}

The goal in the following is to describe a two state quantum system coupled to a bath of quantum mechanical harmonic oscillators. We begin with the case that the bath contains only a single oscillator. Such situation is decribed by the Hamiltonian

$$
\hat{H}_{\mathrm{qo}}^{(s)}=\left(\begin{array}{cc}
\hat{H}_{r}^{(s)} & v \\
v & \hat{H}_{p}^{(s)}+E
\end{array}\right)
$$

where

$$
\begin{aligned}
\hat{H}_{r}^{(s)} & =\frac{\hat{p}^{2}}{2 m}+\frac{1}{2} m \omega^{2} q^{2} \\
\hat{H}_{p}^{(s)} & =\frac{\hat{p}^{2}}{2 m}+\frac{1}{2} m \omega^{2}\left(q-\frac{c}{m \omega^{2}}\right)^{2}
\end{aligned}
$$

denote harmonic oscillator Hamiltonians of the reactant and product states. The additive energy term $E$ denotes here a shift of the zero energy of the product state relative to the reactant state, e.g., denotes the redox energy difference between states $\mathrm{AD}$ and $\mathrm{A}^{-} \mathrm{D}^{+} ; E$ will be considered a variable in the following. If one wishes to describe a process going from the product $\left(\mathrm{A}^{-} \mathrm{D}^{+}\right)$state to the reactant $(\mathrm{AD})$ state the sign of $E$ in (54), representing the redox energy difference, needs to be reversed. This property will be envoked below when we consider both processes, i.e., $\mathrm{AD} \rightarrow \mathrm{A}^{-} \mathrm{D}^{+}$and $\mathrm{A}^{-} \mathrm{D}^{+} \rightarrow \mathrm{AD}$.

The eigenstates and eigenvalues of the Hamiltonians $(55,56)$ are well-known from elementary quantum mechanics; they are

$$
\begin{aligned}
\langle q \mid n\rangle^{(\mathrm{r})} & =\phi_{n}^{(\mathrm{r})}(q)=\left(\frac{\lambda}{\pi}\right)^{\frac{1}{4}}\left(2^{n} n !\right)^{-\frac{1}{2}} H_{n}(\sqrt{\lambda} q) e^{-\frac{1}{2} \lambda q^{2}} \\
\epsilon_{n}^{(\mathrm{r})} & =\hbar \omega\left(n+\frac{1}{2}\right) \\
\langle q \mid n\rangle^{(\mathrm{p})} & =\phi_{n}^{(\mathrm{p})}(q)=\left(\frac{\lambda}{\pi}\right)^{\frac{1}{4}}\left(2^{n} n !\right)^{-\frac{1}{2}} H_{n}\left(\sqrt{\lambda}\left(q-\frac{c}{m \omega^{2}}\right)\right) e^{-\frac{1}{2} \lambda\left(q-c / m \omega^{2}\right)^{2}} \\
\epsilon_{n}^{(\mathrm{p})} & =\hbar \omega\left(n+\frac{1}{2}\right)
\end{aligned}
$$

where

$$
\lambda=m \omega / \hbar
$$

and where $H_{n}(y), n=0,1,2 \ldots$ denote the Hermite polynomials. The reactant states describe an oscillator centered around $q=0$, the product state an oscillator centered around [c.f. (10)]

$$
q_{o}=c / m \omega^{2}
$$


The propagator for the harmonic oscillator is well known. In case of the reactant state the propagator is

$$
\begin{aligned}
\left\langle q^{\prime}\left|e^{-i H_{r}^{(s)}\left(t-t_{o}\right) / \hbar}\right| q\right\rangle= & {\left[\frac{m \omega}{2 i \pi \hbar \sin \omega\left(t-t_{0}\right)}\right]^{\frac{1}{2}} \times } \\
& \exp \left\{\frac{i m \omega}{2 \hbar \sin \omega\left(t-t_{0}\right)}\left[\left(q^{\prime 2}+q^{2}\right) \cos \omega\left(t-t_{0}\right)-2 q^{\prime} q\right]\right\} .
\end{aligned}
$$

This is equivalent to

$$
\begin{aligned}
\left\langle q^{\prime}\left|e^{-i H_{r}^{(s)}\left(t-t_{o}\right) / \hbar}\right| q\right\rangle= & {\left[\frac{\lambda}{2 \pi \sinh \xi}\right]^{\frac{1}{2}} \times } \\
& \exp \left\{-\frac{\lambda}{4}\left[\left(q^{\prime}+q\right)^{2} \tanh \frac{\xi}{2}+\left(q^{\prime}-q\right)^{2} \operatorname{coth} \frac{\xi}{2}\right]\right\} . \\
\xi= & i \omega\left(t-t_{o}\right) .
\end{aligned}
$$

In case of the product state, the same expression applies after replacing $q \rightarrow q-q_{o}$ and $q^{\prime} \rightarrow q^{\prime}-q_{o}$.

The reactant states (57) are occupied in thermal equilibrium with probability

$$
p_{n}^{(\mathrm{r})}=x^{n}(1-x), \quad x=e^{-\hbar \omega / k T},
$$

a result which is well-known from elementary statistical mechanics. The corresponding equilibrium state density matrix of the reactant state oscillator $\rho_{o}^{(\mathrm{r})}$ has the matrix elements

$$
\left[\hat{\rho}_{o}^{(\mathrm{r})}\right]_{m n}=p_{n}^{(\mathrm{r})} \delta_{n m} .
$$

The density matrix can also be written

$$
\hat{\rho}_{o}^{(\mathrm{r})}=2 \sinh (\hbar \omega / 2 k T) e^{-H^{(\mathrm{r})} / k T} .
$$

The transitions from reactant to product states are induced through the matrix elements $v$ in (54). In case of electron transfer in proteins, the coupling is induced through electron tunneling between prosthetic groups in the protein. The corresponding energy values $v$ are very small, usually of the order of $10^{-4} \mathrm{eV}$. As a result, reactant states $|n\rangle^{(\mathrm{r})}$ and product states $|m\rangle^{(\mathrm{p})}$ couple only when they are essentially degenerate. The overall rate of transfer from reactant states $R$ to product states $P$ is then

$$
k_{q o}(R \rightarrow P)=\frac{2 \pi}{\hbar^{2}} v^{2} \mathcal{S}_{q o}(E)
$$

where

$$
\mathcal{S}_{q o}(E)=\left.\left.\sum_{n, m=0}^{\infty} p_{n}^{(\mathrm{r})}\right|^{(\mathrm{r})}\langle n \mid m\rangle^{(\mathrm{p})}\right|^{2} \delta\left(\frac{E+\epsilon_{m}^{(\mathrm{p})}-\epsilon_{n}^{(\mathrm{r})}}{\hbar}\right)
$$


is the so-called spectral line shape function.

We seek to express the line shape function (70) in a more compact form. For this purpose we use the identity

$$
\delta\left(\frac{E+\epsilon_{m}^{(\mathrm{p})}-\epsilon_{n}^{(\mathrm{r})}}{\hbar}\right)=\frac{1}{2 \pi} \int_{-\infty}^{+\infty} d t e^{i t E / \hbar} e^{-i t \epsilon_{n}^{(\mathrm{r})} / \hbar} e^{i t \epsilon_{m}^{(\mathrm{p})} / \hbar} .
$$

Employing the definition of the density matrix (67) one can write (70)

$$
\mathcal{S}_{q o}(E)=\frac{1}{2 \pi} \int_{-\infty}^{+\infty} d t e^{i t E / \hbar} \sum_{n, m=0}^{\infty}{ }^{(\mathrm{r})}\left\langle n\left|\hat{\rho}_{o}^{(\mathrm{r})} e^{-i t \hat{H}_{r}^{(s)} / \hbar}\right| m\right\rangle^{(\mathrm{p})(\mathrm{p})}\left\langle m\left|e^{i t \hat{H}_{p}^{(s)} / \hbar}\right| n\right\rangle^{(\mathrm{r})}
$$

or, equivalently, using (68)

$$
\mathcal{S}_{q o}(E)=\frac{1}{2 \pi} \int_{-\infty}^{+\infty} d t e^{i t E / \hbar} 2 \sinh \frac{\hbar \omega}{2 k T} \operatorname{tr}\left(e^{-\hat{H}_{r}^{(s)} / k T} e^{-i t \hat{H}_{r}^{(s)} / \hbar} e^{i t \hat{H}_{p}^{(s)} / \hbar}\right) .
$$

Expressing the trace as an integral over $q^{\prime}$ we conclude that the spectral line shape function is

$$
\begin{aligned}
& \mathcal{S}_{q o}(E)= \\
& \frac{1}{2 \pi} \int_{-\infty}^{+\infty} d t e^{i t E / \hbar} 2 \sinh \frac{\hbar \omega}{2 k T} \int_{-\infty}^{+\infty} d q \int_{-\infty}^{+\infty} d q^{\prime}\left\langle q^{\prime}\left|e^{-i(t-\hbar / k T) \hat{H}_{r}^{(s)} / \hbar}\right| q\right\rangle\left\langle q e^{i t \hat{H}_{p}^{(s)} / \hbar} \mid q^{\prime}\right\rangle .
\end{aligned}
$$

The propagator (64) allows one to evaluate the line shape function (74). One employs

$$
\begin{aligned}
\left\langle q\left|e^{i t \hat{H}_{p}^{(s)} / \hbar}\right| q^{\prime}\right\rangle= & {\left[\frac{\lambda}{2 \pi \sinh \eta_{1}}\right]^{\frac{1}{2}} \times } \\
& \times \exp \left\{-\frac{\lambda}{4}\left[\left(q^{\prime}+q-2 q_{o}\right)^{2} \tanh \frac{\eta_{1}}{2}+\left(q^{\prime}-q\right)^{2} \operatorname{coth} \frac{\eta_{1}}{2}\right]\right\} . \\
\eta_{1}= & -i \omega t .
\end{aligned}
$$

and, displacing time into the complex plane to account for the equilibrium (temperature $T$ ) density matrix,

$$
\begin{aligned}
\left\langle q^{\prime}\left|e^{-i(t-\hbar / k T) \hat{H}_{r}^{(s)} / \hbar}\right| q\right\rangle= & {\left[\frac{\lambda}{2 \pi \sinh \eta_{2}}\right]^{\frac{1}{2}} \times } \\
& \times \exp \left\{-\frac{\lambda}{4}\left[\left(q^{\prime}+q\right)^{2} \tanh \frac{\eta_{2}}{2}+\left(q^{\prime}-q\right)^{2} \operatorname{coth} \frac{\eta_{2}}{2}\right]\right\} . \\
\eta_{2}= & i \omega t-\hbar \omega / k T .
\end{aligned}
$$

Inserting (75-78) into (74) results in the expression

$$
\mathcal{S}_{q o}(E)=\frac{1}{2 \pi} \int_{-\infty}^{+\infty} d t e^{i t E / \hbar} \frac{\lambda \sinh \left(\frac{\hbar \omega}{2 k T}\right)}{\pi \sqrt{\sinh \eta_{1} \sinh \eta_{2}}} I(t) .
$$


where

$$
\begin{aligned}
I(t) & =\int_{-\infty}^{+\infty} d q \int_{-\infty}^{+\infty} d q^{\prime} \exp \left[-\alpha(t)\left(q+q^{\prime}\right)^{2}-\beta\left(q+q^{\prime}-2 q_{o}\right)^{2}-\gamma\left(q-q^{\prime}\right)^{2}\right] \\
\alpha & =\frac{\lambda}{4} \tanh \frac{\eta_{2}}{2} \\
\beta & =\frac{\lambda}{4} \tanh \frac{\eta_{1}}{2} \\
\gamma & =\frac{\lambda}{4}\left(\tanh \frac{\eta_{1}}{2}+\tanh \frac{\eta_{2}}{2}\right)
\end{aligned}
$$

Expression (79-83) for the spectral line shape function played an important role in the theory of spectral transitions of so-called F-centers in solids as reviewed in [21]. The expression can be further simplified [21]. For this purpose one transforms to new integration variables $u=q+q^{\prime}$ and $u^{\prime}=q-q^{\prime}$. Noting that for the Jacobian holds $\partial\left(u, u^{\prime}\right) / \partial\left(q, q^{\prime}\right)=2$, the integral (80) reads

$$
I(t)=\frac{1}{2} \int_{-\infty}^{+\infty} d u \int_{-\infty}^{+\infty} d u^{\prime} \exp \left[-\alpha(t) u^{2}-\beta\left(u-2 q_{o}\right)^{2}\right] \exp \left[-\gamma u^{\prime 2}\right] .
$$

Completion of the square in the first exponent results in the expression

$$
\begin{aligned}
I(t)= & \frac{1}{2} \exp \left[-4 q_{o}^{2}\left(\beta-\frac{\beta^{2}}{\alpha+\beta}\right)\right] \times \\
& \times \int_{-\infty}^{+\infty} d u^{\prime} \exp \left[-\gamma u^{\prime 2}\right] \int_{-\infty}^{+\infty} d u \exp \left[-(\alpha+\beta)(u-s)^{2}\right] .
\end{aligned}
$$

where

$$
s=2 \beta q_{o} /(\alpha+\beta) .
$$

Since $\operatorname{Re}(\gamma)>0$ and $\operatorname{Re}(\alpha+\beta)>0$ the Gaussian integrals can be evaluated in a straightforward way and one obtains

$$
I(t)=\frac{\pi}{2 \sqrt{\gamma(\alpha+\beta)}} \exp \left[-4 q_{o}^{2}\left(\beta-\frac{\beta^{2}}{\alpha+\beta}\right)\right] .
$$

We note here that this expression and, hence, $\mathcal{S}_{q o}(E)$ do not depend on the sign of $q_{o}$. This is to be expected due to the reflection symmetry of the harmonic oscillator potential. This behaviour implies, however, that a description of a process going from product states to reactant states does not require a change in the sign of $q_{o}$, even though that such change appears to be intuitively necessary.

Using definitions (81-83) and the properties of hyperbolic functions one can show

$$
\frac{\lambda \sinh \left(\frac{\hbar \omega}{2 k T}\right)}{2 \sqrt{\sinh \left(\eta_{1}\right) \sinh \left(\eta_{2}\right) \gamma(\alpha+\beta)}}=1
$$


One can also simplify the exponent in (87). One obtains

$$
\beta-\frac{\beta^{2}}{\alpha+\beta}=\frac{\lambda}{4}\left(\tanh \frac{\eta_{1}}{2}-\frac{\tanh ^{2} \frac{\eta_{1}}{2}}{\tanh \frac{\eta_{1}}{2}+\tanh \frac{\eta_{2}}{2}}\right)=\frac{\lambda}{4} \frac{\tanh \frac{\eta_{1}}{2} \tanh \frac{\eta_{2}}{2}}{\tanh \frac{\eta_{1}}{2}+\tanh \frac{\eta_{2}}{2}} .
$$

Using $\tanh \alpha+\tanh \beta=\sinh (\alpha+\beta) / \cosh \alpha \cosh \beta$ the latter expression can be further rewritten

$$
\begin{aligned}
\beta-\frac{\beta^{2}}{\alpha+\beta} & =\frac{\lambda}{4} \frac{\sinh \frac{\eta_{1}}{2} \sinh \frac{\eta_{2}}{2}}{\sinh \left(\frac{\eta_{1}}{2}+\frac{\eta_{2}}{2}\right)}=\frac{\lambda}{4} \frac{-\sinh \left(\frac{i \omega t}{2}\right) \sinh \left(\frac{i \omega t}{2}+\frac{\hbar \omega}{2 k T}\right)}{\sinh \left(\frac{\hbar \omega}{2 k T}\right)} \\
& =\frac{\lambda}{4} \frac{-\sinh \left(\frac{i \omega t}{2}\right)\left[\sinh \left(\frac{i \omega t}{2}\right) \cosh \left(\frac{\hbar \omega}{2 k T}\right)+\sinh \left(\frac{\hbar \omega}{2 k T}\right) \cosh \left(\frac{i \omega t}{2}\right)\right]}{\sinh \left(\frac{\hbar \omega}{2 k T}\right)} \\
& =\frac{\lambda}{4}\left[\sin ^{2} \frac{\omega t}{2} \operatorname{coth} \frac{\hbar \omega}{2 k T}-i \cos \frac{\omega t}{2} \sin \frac{\omega t}{2}\right]
\end{aligned}
$$

which yields

$$
\beta-\frac{\beta^{2}}{\alpha+\beta}=\frac{\lambda}{8}\left[(1-\cos \omega t) \operatorname{coth} \frac{\hbar \omega}{2 k T}-i \sin \omega t\right]
$$

Combining Eqs. $(62,79,87,88,91)$ results in the final expression

$\mathcal{S}_{q o}(E)=\frac{1}{2 \pi} \int_{-\infty}^{+\infty} d t \exp \left[i t E / \hbar-\frac{c^{2}}{2 m \hbar \omega^{3}} \operatorname{coth} \frac{\hbar \omega}{2 k T}(1-\cos \omega t)+i \frac{c^{2}}{2 m \hbar \omega^{3}} \sin \omega t\right]$

We note here that the rate for the reverse process, i.e., for going from the product state $P$ to the reactant state $R$, is given by

$$
k_{q o}(P \rightarrow R)=\frac{2 \pi}{\hbar^{2}} v^{2} \mathcal{S}_{q o}(-E)
$$

which differs from (69) solely through the sign of $E$.

The integral in (92) can be carried out and the line shape function expressed as a series of regular, modified Bessel functions $I_{k}(x)$ [21]. The result is

$$
\mathcal{S}_{q o}(E)=\frac{e^{-\Lambda\left(1+2 n_{o}\right)}}{\omega}\left(\frac{n_{o}+1}{n_{o}}\right)^{s_{j} / 2} \sum_{k=-\infty}^{\infty} \delta(k-s(E)) I_{k}\left(2 \Lambda \sqrt{n_{o}\left(n_{o}+1\right)}\right)
$$

where $\Lambda=\frac{1}{2} m \omega^{2} q_{o}^{2} / \hbar \omega=c^{2} / 2 m \hbar \omega^{3}$ is the so-called reorganization energy in units of vibrational quanta $\hbar \omega, n_{o}=e^{-\hbar \omega / k T} /\left(1-e^{-\hbar \omega_{j} / k T}\right)$ is the average number of quanta thermally excited in the oscillator, and $s(E)=\left(E-\frac{1}{2} \hbar \omega\right) / \hbar \omega$ counts the number of oscillator levels up to energy $E$. The summation in (93) is over integers $k$ such that one and only one term in the sum contributes anytime that $s(E)$ assumes an integer value. As mentioned above, expression (93) was originally developed to describe optical transitions in solids [21]; it was introduced for the description of thermal electron transfer by Jortner [22, 23]. 


\section{Two State System Coupled to a Multi-Modal Bath of Quantum Mechanical Oscillators}

In case of a two state quantum system coupled to a bath of harmonic oscillators the Hamiltonian (54-56) above is replaced by

$$
\hat{H}_{\mathrm{qb}}=\left(\begin{array}{cc}
\hat{H}_{\mathrm{r}} & v \\
v & \hat{H}_{\mathrm{p}}+E
\end{array}\right)
$$

where

$$
\begin{aligned}
& \hat{H}_{r}=\sum_{j}\left(\frac{\hat{p}_{j}^{2}}{2 M_{j}}+\frac{1}{2} M_{j} \omega_{j}^{2} q_{j}^{2}\right) \\
& \hat{H}_{p}=\sum_{j}\left(\frac{\hat{p}_{j}^{2}}{2 M_{j}}+\frac{1}{2} M_{j} \omega_{j}^{2}\left(q_{j}-\frac{c_{j}}{M_{j} \omega_{j}^{2}}\right)^{2}\right)
\end{aligned}
$$

The Hamiltonians $\hat{H}_{r}$ and $\hat{H}_{p}$ describe here the same situation as in case of the Hamiltonian (54-56), except that the coupling is to an ensemble of oscillators which represent the protein matrix. One can absorb the masses $M_{j}$ in the definition of the positions $q_{j}$ of the oscillators such that we assume in the following

$$
M_{j}=1 \quad \forall j
$$

The eigenstates of the Hamiltonians $(96,97)$ are denoted by $\langle\vec{q} \mid \vec{n}\rangle^{(\mathrm{r})}$ and $\langle\vec{q} \mid \vec{m}\rangle^{(\mathrm{p})}$, respectively, where $\vec{q}=\left(q_{1}, q_{2}, \ldots\right)^{T}$ collects the coordinates describing the protein matrix and where $\vec{n}=\left(n_{1}, n_{2}, \ldots\right)$ denotes the set of quantum numbers $n_{j}$ of the individual oscillators. We assume that all degrees of freedom of the protein matrix are harmonic. The eigenstates and eigenvalues are explicitly

$$
\begin{aligned}
\langle\vec{q} \mid \vec{n}\rangle^{(\mathrm{r})} & =\prod_{j}\left\langle q_{j} \mid n_{j}\right\rangle^{(\mathrm{r}, j)}=\prod_{j}\left(\frac{\lambda_{j}}{\pi}\right)^{\frac{1}{4}}\left(2_{j}^{n} n_{j} !\right)^{-\frac{1}{2}} H_{m_{j}}\left(\sqrt{\lambda_{j}} q_{j}\right) e^{-\frac{1}{2} \lambda q_{j}^{2}} \\
\epsilon_{\vec{n}}^{(\mathrm{r}, j)} & =\sum_{j} \hbar \omega_{j}\left(n_{j}+\frac{1}{2}\right) \\
\langle\vec{q} \mid \vec{m}\rangle^{(\mathrm{p})} & =\prod_{j}\left\langle q_{j} \mid m_{j}\right\rangle^{(\mathrm{p}, j)} \\
& =\prod_{j}\left(\frac{\lambda_{j}}{\pi}\right)^{\frac{1}{4}}\left(2_{j}^{m} m_{j} !\right)^{-\frac{1}{2}} H_{m_{j}}\left(\sqrt{\lambda_{j}}\left(q_{j}-\frac{c_{j}}{\omega_{j}^{2}}\right)\right) e^{-\frac{1}{2} \lambda_{j}\left(q_{j}-c_{j} / \omega_{j}^{2}\right)^{2}} \\
\epsilon_{n}^{(\mathrm{p}, j)} & =\sum_{j} \hbar \omega_{j}\left(m_{j}+\frac{1}{2}\right)
\end{aligned}
$$

where

$$
\lambda_{j}=\omega_{j} / \hbar
$$


The overall rate of transfer from reactant states to product states can be written again in the form

$$
k_{q b}(R \rightarrow P)=\frac{2 \pi}{\hbar^{2}} v^{2} \mathcal{S}_{q o}(E)
$$

where, according to $(70,71)$, the line shape function is

$$
\mathcal{S}_{q b}(E)=\frac{1}{2 \pi} \int_{-\infty}^{+\infty} d t e^{i t E / \hbar} \prod_{j}\left(\left.\left.\sum_{n_{j}, m_{j}=0}^{\infty} p_{n_{j}}^{(\mathrm{r}, \mathrm{j})} e^{-i t \epsilon_{n_{j}}^{(\mathrm{r}, j)} / \hbar}\right|^{(\mathrm{r}, j)}\langle n \mid m\rangle^{(\mathrm{p}, j)}\right|^{2} e^{i t \epsilon_{m_{j}}^{(\mathrm{p}, j)} / \hbar}\right) .
$$

with

$$
p_{n}^{(\mathrm{r}, j)}=x_{j}^{n}\left(1-x_{j}\right), \quad x_{j}=e^{-\hbar \omega_{j} / k T},
$$

Each of the factors on the r.h.s. of (105) corresponds to the single respective term in (72), i.e., to a term which has been evaluated above [c.f. (92)]. Comparision with (92) allows one then to evaluate (105) as well. This leads to the expression $[24,25,26]$

$$
k_{q b}(R \rightarrow P)=\frac{v^{2}}{\hbar^{2}} \int_{-\infty}^{+\infty} d t e^{i t E / \hbar} e^{i Q_{1}(t) / \pi \hbar} e^{-Q_{2}(t) / \pi \hbar}
$$

where

$$
\begin{aligned}
Q_{1}(t) & =\frac{\pi}{2} \sum_{j} \frac{c_{j}^{2}}{\hbar \omega_{j}^{3}} \sin \omega_{j} t \\
Q_{2}(t) & =\frac{\pi}{2} \sum_{j} \frac{c_{j}^{2}}{\hbar \omega_{j}^{3}} \operatorname{coth} \frac{\hbar \omega_{j}}{2 k T}\left[1-\cos \left(\omega_{j} t\right)\right] .
\end{aligned}
$$

One can express the rate for the backward reaction by reversing the sign of $E$ in this expression [26]

$$
k_{q b}(P \rightarrow R)=\frac{v^{2}}{\hbar^{2}} \int_{-\infty}^{+\infty} d t e^{-i t E / \hbar} e^{i Q_{1}(t) / \pi \hbar} e^{-Q_{2}(t) / \pi \hbar} .
$$

A kinetic system with two states $R$ and $P$ is described by the vector $\vec{p}(t)=$ $\left(p_{R}(t), p_{P}(t)\right)^{T}$ where the components $p_{R}(t)$ and $p_{P}(t)$ account for the probability to observe, at time $t$, the states $R$ and $P$, respectively. $\vec{p}(t)$ obeys the rate equation

$$
\frac{d}{d t}\left(\begin{array}{c}
p_{R}(t) \\
p_{P}(t)
\end{array}\right)=\left(\begin{array}{cc}
-k_{q b}(R \rightarrow P) & k_{q b}(P \rightarrow R) \\
k_{q b}(R \rightarrow P) & -k_{q b}(P \rightarrow R)
\end{array}\right)\left(\begin{array}{c}
p_{R}(t) \\
p_{P}(t)
\end{array}\right)
$$

which needs to be solved for some initial condition. In the present case we consider a system starting in state $R$, i.e., the initial condition is $\vec{p}(0)=(1,0)^{T}$. The corresponding solution of (111) is

$$
\begin{aligned}
\left(\begin{array}{c}
p_{R}(t) \\
p_{P}(t)
\end{array}\right)= & \frac{1}{k_{q b}(R \rightarrow P)+k_{q b}(P \rightarrow R)}\left[\left(\begin{array}{c}
k_{q b}(P \rightarrow R) \\
k_{q b}(R \rightarrow P)
\end{array}\right)+\right. \\
& \left.+\left(\begin{array}{r}
k_{q b}(R \rightarrow P) \\
-k_{q b}(R \rightarrow P)
\end{array}\right) \exp \left\{-\left[k_{q b}(R \rightarrow P)+k_{q b}(P \rightarrow R)\right]\right\}\right] .
\end{aligned}
$$


Accordingly, the state $R$ decays with a rate $k_{q b}(R \rightarrow P)+k_{q b}(P \rightarrow R)$ to an equilibrium mixture of the states $R$ and $P$, i.e., the relaxation rate $k_{\text {rel }}$ of the initial state $R$, according to $(107,110)$ is

$$
k_{\mathrm{rel}}=2 \frac{v^{2}}{\hbar^{2}} \int_{-\infty}^{+\infty} d t \cos (-i t E / \hbar) e^{i Q_{1}(t) / \pi \hbar} e^{-Q_{2}(t) / \pi \hbar} .
$$

Since $Q_{1}(t)$, according to (108), is an odd function of $t$ we note that in

$$
e^{i Q_{1}(t) / \pi \hbar}=\cos \left(Q_{1}(t) / \pi \hbar\right)+i \sin \left(Q_{1}(t) / \pi \hbar\right)
$$

the real part is an even function of $t$ and the imaginary part is an odd function of $t$. Since, according to (109) $Q_{2}(t)$ and, hence, also $\exp \left(-Q_{2}(t) / \pi \hbar\right)$ is an even function of $t$, the time integral involving $\sin \left(Q_{1}(t) / \pi \hbar\right)$ vanishes and one is left with the expression [26]

$$
k_{\mathrm{rel}}=\frac{2 v^{2}}{\hbar^{2}} \int_{0}^{+\infty} d t \cos (-i t E / \hbar) \cos \left(Q_{1}(t) / \pi \hbar\right) e^{-Q_{2}(t) / \pi \hbar}
$$

The result obtained, at first sight, appears to be of little value since evaluation of the functions $Q_{1}(t)$ and $Q_{2}(t)$, according to $(108,109)$, requires knowledge of the quantities $c_{j}^{2} / \hbar \omega_{j}^{3}$ for the ensemble of oscillators. Fortunately, it is possible to achieve the evaluation in case that the spectral density

$$
J(\omega)=\frac{\pi}{2} \sum_{j} \frac{c_{j}^{2}}{\omega_{j}} \delta\left(\omega-\omega_{j}\right)
$$

is known. In fact, one can express

$$
\begin{aligned}
Q_{1}(t) & =\int_{0}^{\infty} d \omega \omega^{-2} J(\omega) \sin \omega t \\
Q_{2}(t) & =\frac{\pi}{2} \int_{0}^{\infty} d \omega \omega^{-2} J(\omega) \operatorname{coth} \frac{\hbar \omega}{2 k T}(1-\cos \omega t) .
\end{aligned}
$$

We want to demonstrate in the next section that the density $J(\omega)$ can be determined from molecular dynamics simulations in which the function $\epsilon(t)=\Delta E[\mathbf{R}(t)]$, as defined in (29), is monitored. In fact, $J(\omega)$ is the spectral density of $\Delta E[\mathbf{R}(t)]$.

\section{From the Energy Gap Correlation Function $\Delta E[\mathbf{R}(t)]$ to the Spectral Density $J(\omega)[2]$}

In this section we will derive a relationship which connects, in the limit of high temperature, $J(\omega)$ and the auto-correlation function $C_{\epsilon \epsilon}(t)$ of the energy gap function, introduced in Section 4. We start from definition (39) of $C_{\epsilon \epsilon}(t)$ and express, according to $(97)$,

$$
\epsilon(t)=\hat{H}_{p}-\hat{H}_{r}+E
$$


where the Hamiltonian operators will be interpreted as Hamiltonian functions of classical bath oscillators, an identification which holds at high temperatures. One obtains

$$
\epsilon(t)=-\sum_{j} c_{j} q_{j}(t)+\sum_{j} \frac{1}{2} \omega_{j}^{-2} c_{j}^{2} .
$$

The time average of $\epsilon(t)$ in the reactant state, i.e., for $\left\langle q_{j}\right\rangle_{R}=0$, is

$$
\langle\epsilon\rangle_{R}=\sum_{j} \frac{1}{2} \omega_{j}^{-2} c_{j}^{2}
$$

In the product state holds $\left\langle q_{j}\right\rangle_{P}=c_{j} / \omega_{j}$ and, hence, the corresponding average of $\epsilon(t)$ is

$$
\langle\epsilon\rangle_{P}=-\sum_{j} \frac{1}{2} \omega_{j}^{-2} c_{j}^{2}+E
$$

We want to evaluate now the equilibrium correlation function $C_{\epsilon \epsilon}(t)$ for the protein in the reactant state $R$. For this purpose we start from the expression

$$
C_{\epsilon \epsilon}(t)=\frac{\langle(\epsilon(t)-\langle\epsilon\rangle)(\langle\epsilon(0)-\langle\epsilon\rangle)\rangle}{\langle\epsilon(0)-\langle\epsilon\rangle\rangle^{2}}
$$

The quantity $\delta \epsilon(t)=\epsilon(t)-\langle\epsilon\rangle$ which enters here is, using (121),

$$
\delta E(t)=-\sum_{j} c_{j} q_{j}(t) .
$$

In the high temperature limit the motion of the bath oscillators is described by

$$
q_{j}(t)=A_{j} \cos \left(\omega_{j} t+\varphi_{j}\right) .
$$

The numerator $C_{1}(t)$ of the r.h.s. of (123) can then be written

$$
\begin{aligned}
C_{1}(t) & =\langle\delta \epsilon(t) \delta \epsilon(o)\rangle \\
& =\left\langle\left(\sum_{j} c_{j} A_{j} \cos \left(\omega_{j} t+\varphi_{j}\right)\right)\left(\sum_{j}^{\prime} c_{j^{\prime}} A_{j^{\prime}} \cos \varphi_{j^{\prime}}\right)\right\rangle \\
& =\frac{1}{4}\left\langle\sum _ { j , j ^ { \prime } } c _ { j } c _ { j ^ { \prime } } A _ { j } A _ { j ^ { \prime } } \left[\exp \left(i\left(\omega_{j} t+\varphi_{j}+\varphi_{j^{\prime}}\right)\right)+\right.\right. \\
& +\exp \left(-i\left(\omega_{j} t+\varphi_{j}+\varphi_{j^{\prime}}\right)\right)+\exp \left(i\left(\omega_{j} t+\varphi_{j}-\varphi_{j^{\prime}}\right)\right)+ \\
& \left.\left.+\exp \left(-i\left(\omega_{j} t+\varphi_{j}-\varphi_{j^{\prime}}\right)\right)\right]\right\rangle
\end{aligned}
$$

where the initial phases $\varphi_{j}$ and $\varphi_{j^{\prime}}$ are uniformly distributed in the interval $[0,2 \pi)$.

In carrying out the averages in (126) we follow Rayleigh [27, 28] and note that for the averages over the phases holds

$$
\left\langle\exp \left(i\left(\varphi_{j}+\varphi_{j^{\prime}}\right)\right)\right\rangle=0 ; \quad\left\langle\exp \left(i\left(\varphi_{j}-\varphi_{j^{\prime}}\right)\right)\right\rangle=\delta_{j j^{\prime}} .
$$


This yields

$$
C_{1}(t)=\frac{1}{2} \sum_{j} c_{j}^{2}\left\langle A_{j}^{2}\right\rangle \cos \omega_{j} t .
$$

Using the well-known fact that the average energy of a harmonic oscillator is $k_{B} T$, i.e.,

$$
\frac{1}{2} \omega_{j}^{2}\left\langle A_{j}^{2}\right\rangle=k_{B} T,
$$

one concludes

$$
C_{1}(t)=\sum_{j=1}^{N} c_{j}^{2} \frac{k_{B} T}{\omega_{j}^{2}} \cos \omega_{j} t .
$$

This can be expressed, employing the definition (116) of $J(\omega)$,

$$
C_{1}(t)=\frac{2 k_{B} T}{\pi} \int_{0}^{\infty} \mathrm{d} \omega \frac{J(\omega)}{\omega} \cos \omega t .
$$

Noting $C(t)=C_{1}(t) / C_{1}(0)$ one obtains finally

$$
C_{\epsilon \epsilon}(t)=\frac{\int_{0}^{\infty} \mathrm{d} \omega J(\omega) / \omega \cos \omega t}{\int_{0}^{\infty} \mathrm{d} \omega J(\omega) / \omega},
$$

a relationship which has been stated before [29, 30].

We want to express $J(\omega)$ through $C_{\epsilon \epsilon}(t)$. Fourier's theorem yields

$$
\frac{2}{\pi} \int_{0}^{\infty} d t \cos \omega t\left[\int_{0}^{\infty} d \omega^{\prime} \frac{J\left(\omega^{\prime}\right)}{\omega^{\prime}} \cos \omega^{\prime} t\right]=\frac{J(\omega)}{\omega}
$$

and, hence, one can state

$$
\frac{J(\omega)}{\omega}=\frac{2}{\pi}\left[\int_{0}^{\infty} d \omega \frac{J(\omega)}{\omega}\right] \int_{0}^{\infty} d t C_{\epsilon \epsilon}(t) \cos \omega t .
$$

The integral $\int_{o}^{\infty} d \omega J(\omega) / \omega$ can be expressed in terms of the variance $\sigma^{2}=\left\langle\epsilon^{2}\right\rangle-$ $\langle\epsilon\rangle^{2}$. For this purpose we consider first a subset of $N_{j}$ bath oscillators with identical frequencies $\omega_{j}$. We designate these oscillators by a second (beside $j$ ) index $\alpha . \delta E(t)$, according to (124), is

$$
\delta E(t)=-\sum_{j} \sum_{\alpha} c_{j \alpha} A_{j \alpha} \cos \left(\omega_{j} t+\varphi_{j \alpha}\right) .
$$

In this expression appear the quantities

$$
\begin{aligned}
U_{j \alpha} & =c_{j \alpha} A_{j \alpha} \cos \left(\omega_{j} t+\varphi_{j \alpha}\right) \\
U_{j} & =\sum_{\alpha} U_{j \alpha}
\end{aligned}
$$


which will be characterized further; specifically, we seek to determine the distribution of $U_{j}$.

The distribution of the quantity (137) for oscillators in thermal equilibrium has been provided by Rayleigh [27, 28]. Following his procedure one notes

$$
\begin{aligned}
U_{j} & =\sum_{\alpha} c_{j \alpha} A_{j \alpha} \cos \left(\omega_{j} t+\varphi_{j \alpha}\right) \\
& =\cos \left(\omega_{j} t\right) \sum_{\alpha} c_{j \alpha} A_{j \alpha} \cos \varphi_{j \alpha}-\sin \left(\omega_{j} t\right) \sum_{\alpha} c_{j \alpha} A_{j \alpha} \sin \varphi_{j \alpha} \\
& =\gamma_{j} \cos \left(\omega_{j} t+\vartheta_{j}\right)
\end{aligned}
$$

where

$$
\begin{aligned}
\gamma_{j} & =\sqrt{\left(\sum_{\alpha} c_{j \alpha} A_{j \alpha} \cos \varphi_{j \alpha}\right)^{2}+\left(\sum_{\alpha} c_{j \alpha} A_{j \alpha} \sin \varphi_{j \alpha}\right)^{2}} \\
\tan \vartheta_{j} & =\left(\sum_{\alpha} c_{j \alpha} A_{j \alpha} \sin \varphi_{j \alpha}\right) /\left(\sum_{\alpha} c_{j \alpha} A_{j \alpha} \cos \varphi_{j \alpha}\right) .
\end{aligned}
$$

Rayleigh has shown [28] that $\gamma_{j}$ and $\vartheta_{j}$ are randomly distributed for large $N_{j}$. The distribution $p_{j}\left(\gamma_{j}\right)$ of $\gamma_{j}$ is

$$
p_{j}\left(\gamma_{j}\right)=\frac{2}{\delta_{j}^{2}} \gamma_{j} \exp \left(-\frac{\gamma_{j}^{2}}{\delta_{j}^{2}}\right), \quad \delta_{j}=\sum_{\alpha} c_{j \alpha}^{2} A_{j \alpha}^{2} .
$$

The distribution of the phase angles $\vartheta_{j}$ is immaterial as the derivation below will show. According to the above calculations $U_{j}$ behaves like a single oscillator with randomly distributed amplitudes $\gamma_{j}$ and randomly distributed phases $\vartheta_{j}$.

Let us consider now a particular oscillator of this ensemble, described by fixed $\gamma_{j}$ and $\vartheta_{j}$,

$$
U_{j}(t)=\gamma_{j} \cos \left(\omega_{j} t+\vartheta_{j}\right)
$$

Sampling such $U_{j}(t)$ at many time points $t=t_{1}, t_{2}, \ldots$ leads to a distribution [31]

$$
\hat{p}_{j}\left(U_{j}\right)= \begin{cases}\frac{1}{\pi \sqrt{\gamma_{j}^{2}-U_{j}^{2}}} & \text { for } U_{j} \leq \gamma_{j} \\ 0 & \text { for } U_{j}>\gamma_{j} .\end{cases}
$$

For a large ensemble of oscillators with a distribution of $\gamma_{j}$ and $\vartheta_{j}$ values as described above the distribution of $U_{j}$ can be determined now in a straightforward way. For the oscillator (142) all ensemble elements with $\gamma_{j} \geq U_{j}$ contribute. The distribution $\tilde{p}\left(U_{j}\right)$ for the whole ensemble of bath oscillators is, therefore,

$$
\begin{aligned}
\tilde{p}\left(U_{j}\right) & =\int_{U_{j}}^{\infty} d \gamma_{j} p_{j}\left(\gamma_{j}\right) \hat{p}_{j}\left(U_{j}\right) \\
& =\int_{U_{j}}^{\infty} d \gamma_{j} \frac{2}{\delta_{j}^{2}} \gamma_{j} \exp \left(-\frac{\gamma_{j}^{2}}{\delta_{j}^{2}}\right) \frac{1}{\pi \sqrt{\gamma_{j}^{2}-U_{j}^{2}}}
\end{aligned}
$$


The integral can be evaluated analytically. For this purpose we introduce the variable $y=\gamma_{j}^{2}-U_{j}^{2}$. Using

$$
\int_{0}^{\infty} d y \frac{1}{\sqrt{y}} e^{-\lambda y}=\sqrt{\frac{\pi}{\lambda}}
$$

one obtains

$$
\tilde{p}\left(U_{j}\right)=\frac{1}{\sqrt{\pi \tilde{\sigma}^{2}}} \exp \left(-\frac{U_{j}^{2}}{\tilde{\sigma}_{j}^{2}}\right),
$$

i.e., a Gaussian distribution.

According to (135-137) holds $\delta E=\sum_{j} U_{j}$. Since each of the terms in this sum is Gaussian-distributed, the distribution of $\delta E$ is Gaussian as well, namely,

$$
\begin{aligned}
p(\delta E) & =\frac{1}{2 \pi \sigma^{2}} \exp \left(-\frac{(\delta E)^{2}}{2 \sigma^{2}}\right) \\
\sigma^{2} & =\frac{1}{2} \sum_{j} \sigma_{j}^{2}=\frac{1}{2} \sum_{j, \alpha} c_{j \alpha}^{2} A_{j \alpha}^{2} .
\end{aligned}
$$

In the classical, i.e., high temperature, limit holds at equilibrium

$$
\sigma^{2}=\frac{1}{2} \sum_{j, \alpha} c_{j \alpha}^{2}\left\langle A_{j \alpha}^{2}\right\rangle=\frac{1}{2} \sum_{j, \alpha} \frac{2 k_{B} T^{2}}{\omega_{j}} c_{j \alpha}^{2}
$$

where we have used (129). One can then express the sum over the coefficients $c_{j \alpha}^{2}$ through $J(\omega)$ using the definition of the latter, i.e., (116), and, hence, one can conclude

$$
\sigma^{2}=\frac{2 k_{B} T}{\pi} \int_{0}^{\infty} d \omega \frac{J(\omega)}{\omega} .
$$

We can finally provide the desired expression of $J(\omega)$. Employing (150) in (134) yields

$$
\frac{J(\omega)}{\omega}=\frac{\sigma^{2}}{k_{B} T} \int_{0}^{\infty} d t C(t) \cos \omega t .
$$

Accordingly, $J(\omega)$ can be determined from a molecular dynamics simulation which records the fluctuations of $\epsilon(t)=\Delta E[\mathbf{R}(t)]$ and determines then the energy gap correlation function (123). In case that the gap correlation function obeys a simple exponential decay (41) one can evaluate (151) further and obtains

$$
\frac{J(\omega)}{\omega}=\frac{\eta \omega}{1+\omega^{2} \tau^{2}}, \quad \eta=\frac{\sigma^{2} \tau}{k_{B} T},
$$

a form of $J(\omega)$ known as the Debey function. 


\section{Evaluating the Transfer Rate[2, 3]}

We want to discuss now the evaluation of the relaxation rate $k_{\text {rel }}$. The evaluation is based on Eqs. $(115,117,118)$ and requires knowledge of the spectral density $J(\omega)$ given by (152). For this purpose the material properties $\sigma, \tau$ and $\langle\epsilon\rangle$ are required as determined through a molecular dynamics simulation. Typical values of $\sigma$ and $\tau$ are $3.9 \mathrm{kcal} / \mathrm{mol}$ and $94 \mathrm{fs}$, determined for electron transfer at $T=300 \mathrm{~K}$ in the photosynthetic reaction center in Rhodopseudomonas viridis [1]. The value of $\langle\epsilon\rangle$ is difficult to determine precisely and one may rather determine the rate $k_{\text {rel }}$ as a function of $E$ and shift $k_{\text {rel }}(E)$ along the energy axis according to available experimental information. $k_{\text {rel }}(E)$, at physiological temperatures, assumes a maximum value for a given $E$-value and one may argue that in a given protein the rate process has been

optimized through adapting the additive contributions $\langle\epsilon\rangle$ and redox energy difference E.

In case of $J(\omega)$, given by the Debye function (152), one can determine $Q_{1}(t)$ defined in (117)

$$
Q_{1}(t)=\int_{0}^{\infty} d \omega \frac{\eta \sin \omega t}{\omega\left(1+\omega^{2} \tau^{2}\right)}=\frac{\eta \pi}{2}\left[1-\exp \left(-\frac{t}{\tau}\right)\right]
$$

Using $1-\cos \omega t=2 \sin ^{2}(\omega t / 2)$ one can write $(115)$

$$
\begin{aligned}
k_{\mathrm{rel}}(E, T)= & \left(\frac{2 v}{\hbar}\right)^{2} \int_{0}^{\infty} d t \cos \left(\frac{E t}{\hbar}\right) \cos \left[\frac{\eta}{2 \hbar}\left(1-e^{-t / \tau}\right)\right] \times \\
& \times \exp \left[-\frac{2 \eta}{\pi \hbar} \int_{0}^{\infty} d \omega \frac{\sin ^{2}\left(\frac{\omega t}{2}\right)}{\omega\left(1+\omega^{2} \tau^{2}\right)} \operatorname{coth}\left(\frac{\beta \hbar \omega}{2}\right)\right] .
\end{aligned}
$$

To simplify this expression we define $x=t / \tau, y=\omega \tau$, and $\gamma=\eta / h$ which yields

$$
\begin{aligned}
k_{\text {rel }}(E, T)= & \left(\frac{2 V}{\hbar}\right)^{2} \tau \int_{0}^{\infty} d x \cos \left(\frac{E \tau}{\hbar} x\right) \cos \left[\gamma \pi\left(1-e^{-x}\right)\right] \times \\
& \times \exp \left[-4 \gamma \int_{0}^{\infty} d y \frac{\sin ^{2}\left(\frac{x y}{2}\right)}{y\left(1+y^{2}\right)} \operatorname{coth}\left(\frac{\hbar}{2 k_{B} \tau} \cdot \frac{y}{T}\right)\right]
\end{aligned}
$$

A typical numerical value for $V / \hbar$ is $5 \mathrm{ps}^{-1}[1]$.

A straightforward numerical evaluation of (155) is time consuming since it involves a double integral. One can use a more convenient, albeit approximate, expression for the $\exp [\cdots]$ factor in the integrand of $(155)$. We define for this purpose

$$
q_{2}(x)=\int_{0}^{\infty} d y \frac{\sin ^{2}\left(\frac{x y}{2}\right)}{y\left(1+y^{2}\right)} \operatorname{coth}(\alpha y), \quad \alpha=\frac{\hbar}{2 k_{B} \tau T}
$$


As demonstrated in [2], $q_{2}(x)$ is a monotonously increasing function of $x$. The main contribution to (156) stems from the region of small $x$. One may employ an expansion of $q_{2}(x)$ which holds for small $x$

$$
q_{2}(x) \approx \frac{x^{2}}{4}[f(\alpha)-\ln x]
$$

where

$$
f(\alpha)=\int_{0}^{\infty} \frac{y d y}{1+y^{2}}[\operatorname{coth}(\alpha y)-1] .
$$

Since $q_{2}(x)$ is $E$-independent one can use the same numerical approximation for all $E$-values considered. Hence, for a given temperature obtaining $k(E, T)$ at all different $E$ values requires one to evaluate $q_{2}(x)$ only once. Then (155) is evaluated using

$$
k_{\text {appr }}(E, T)=\left(\frac{2 V}{\hbar}\right)^{2} \tau \int_{0}^{\infty} d x \cos \left(\frac{E \tau}{\hbar} x\right) \cos \left[\gamma \pi\left(1-e^{-x}\right)\right] e^{-4 \gamma q_{2}(x)}
$$

with $q_{2}(x)$ given by $(157,157)$.

High Temperature Limit In the limit of high temperature the expression (115) of the electron transfer rate can be evaluated by the method of steepest descent. This approximation is based on a quadratic expansion of $Q_{2}(t)$ around its minimum at $t=0$. The procedure requires one to determine the quantity

$$
\mu=\left.\frac{d^{2}}{d t^{2}} Q_{2}(t)\right|_{t=0}
$$

The expression for $Q_{2}(t)$ in (154) yields

$$
\mu=\int_{0}^{\infty} d \omega J(\omega) \operatorname{coth}\left(\frac{\beta \hbar \omega}{2}\right) .
$$

Unfortunately, for many choices of $J(\omega)$ this expression diverges and the steepest descent method cannot be applied. However, we note that the divergence of (161) is due to $\omega \rightarrow \infty$ contributions to the integral over $J(\omega)$. Since the number of modes in a protein are finite, the divergence in (161) is due to an artificial analytical form of $J(\omega)$. If one would assume a cut-off frequency $\omega_{c}$, i.e., replace $J(\omega)$ by $J(\omega) \theta\left(\omega-\omega_{c}\right)$, a divergence would not arise in (161). One may, hence, assume that the second derivative (160) actually exists, approximate

$$
Q_{2}(t) \approx \frac{1}{2} \mu t^{2}
$$

and employ this in a steepest descent method. 
At a sufficiently high temperature, contributions to the integral in (115) arise only in a vicinity of $t=0$ where $(162)$ is small. In this case, one can approximate $Q_{1}(t)$ in (154) linearly around $t=0$

$$
Q_{1}(t) \approx \nu t ; \quad \nu=\left.\frac{d}{d t} Q_{1}(t)\right|_{t=0}
$$

where

$$
\nu=\int_{0}^{\infty} d \omega \frac{J(\omega)}{\omega} .
$$

By using the approximations (162) and (163) in (115), if $E$ is not close to 0, one obtains $[32,33,2]$

$$
k_{\mathrm{rel}}(E, T) \approx \frac{2 \pi V^{2}}{\hbar} \frac{1}{\sqrt{2 \pi \delta^{2}}} \exp \left[-\frac{\left(E-E_{m}\right)^{2}}{2 \delta^{2}}\right] .
$$

where

$$
\begin{aligned}
\delta^{2} & =\frac{\hbar \mu}{\pi}=\frac{\hbar}{\pi} \int_{0}^{\infty} d \omega J(\omega) \operatorname{coth}\left(\frac{\beta \hbar \omega}{2}\right) \\
E_{m} & =\frac{\nu}{\pi}=\frac{1}{\pi} \int_{0}^{\infty} d \omega \frac{J(\omega)}{\omega} .
\end{aligned}
$$

At a high enough temperature, i.e., for $T>100 \mathrm{~K}$, according to our numerical calculations, one can show further [2]

$$
\delta=\sigma ; \quad E_{m}=\frac{\sigma^{2}}{2 k_{B} T}
$$

According to (167), $E_{m}$ is actually temperature independent. Hence, one can rewrite (165) into a form which agrees with the rate as given by the classical Marcus theory

$$
k_{M}(\epsilon, T)=\frac{2 \pi V^{2}}{\hbar} \frac{1}{\sqrt{2 \pi f k_{B} T q_{o}^{2}}} \exp \left[-\frac{\left(\epsilon-\frac{1}{2} f q_{o}^{2}\right)^{2}}{2 k_{B} T f q_{o}^{2}}\right]
$$

where

$$
f q_{o}^{2}=2 \epsilon_{m}=\left.\frac{\sigma^{2}}{k_{B} T}\right|_{T=300 K} .
$$

Low Temperature Limit At low temperatures, one can employ (158) for $\alpha=$ $\hbar / 2 \tau k_{B} T \rightarrow \infty$, to approximate $q_{2}(x)$ further. It can be verified

$$
\lim _{\alpha \rightarrow \infty} f(\alpha)=\frac{\pi^{2}}{12 \alpha^{2}}
$$


The value of the integral in (159) results mainly from contributions at small x. Accordingly, at low temperatures one can assume the overall integrand to be dominated by the interval in which $\gamma \pi^{2} x^{2} / 12 \alpha^{2}$ is small. Therefore, one can apply (157) to expand the exponential part of (159),

$$
\begin{aligned}
e^{-4 \gamma q_{2}(x)} & =\exp \left(\gamma x^{2} \ln x-\frac{\gamma \pi^{2} x^{2}}{12 \alpha^{2}}\right) \\
& =\exp \left(\gamma x^{2} \ln x\right)\left[1-\left(\frac{\gamma \pi^{2} x^{2}}{12}\right)\left(\frac{2 k_{B} \tau T}{\hbar}\right)^{2}\right] .
\end{aligned}
$$

Then the electron transfer rate at $T \rightarrow 0$ can be expressed

$$
k(E, T) \approx k(E, 0)-k_{1}(E)\left(\frac{2 k_{B} \tau T}{\hbar}\right)^{2},
$$

where

$k_{1}(E)=\left(\frac{2 V}{\hbar}\right)^{2} \tau \int_{0}^{\infty} d x \cos \left(\frac{E \tau}{\hbar} x\right) \cos \left[\gamma \pi\left(1-e^{-x}\right)\right]\left(-\frac{\gamma \pi^{2} x^{2}}{12}\right) \exp \left(\gamma x^{2} \ln x\right)$.

From (173), one concludes that at low temperatures, the electron transfer rate is actually changing very slowly with temperature. This behavior has been found in many observations $[34,35]$.

Results In Fig. 2 we present calculated electron transfer rates $k_{\text {rel }}(E, T)$ as a function of the redox energy difference $E$ for temperatures $T=10 \mathrm{~K}$ and $T=300 \mathrm{~K}$, and compare the results to transfer rates predicted by the Marcus theory, e.g., by Eq. $(169,170)$. One can observe that at physiological temperatures, the rate evaluated from the Marcus theory in a wide range of $E$-values, agrees well with the rate evaluated from the spin-boson model at $T=300 \mathrm{~K}$, a behavior which is expected from the high temperature limit derived above. However the Marcus theory and the spin-boson model differ significantly at $T=10 \mathrm{~K}$. At such low temperature the rate as a function of $E$ for the spin-boson model is asymmetrical. This result agrees with observations reported in [36] which show a distinct asymmetry with respect to $E_{m}$ at low temperatures. Such asymmetry is not predicted by the models of Marcus and Hopfield [37, 38, 39].

If one makes the assumption that biological electron transfer systems evolved their $E$-values such that rates are optimized, one should expect that electron transfer rates in the photosynthetic reaction center are formed through a choice of $E \rightarrow E_{\max }$, such that $k\left(E_{\text {max }}\right)$ is a maximum. In Fig. 3 we present corresponding maximum transfer rates, $k\left(E_{\max }\right)$ as well as $k(E, T)$ for non-optimal values of $E=E_{m} \pm \delta$, where $\delta=$ $2.5 \mathrm{kcal} / \mathrm{mol}$. Experimental data of electron transfer processes in the photosynthetic reaction center show increases similarly to those presented in Fig. 3 [40, 41, 42, 


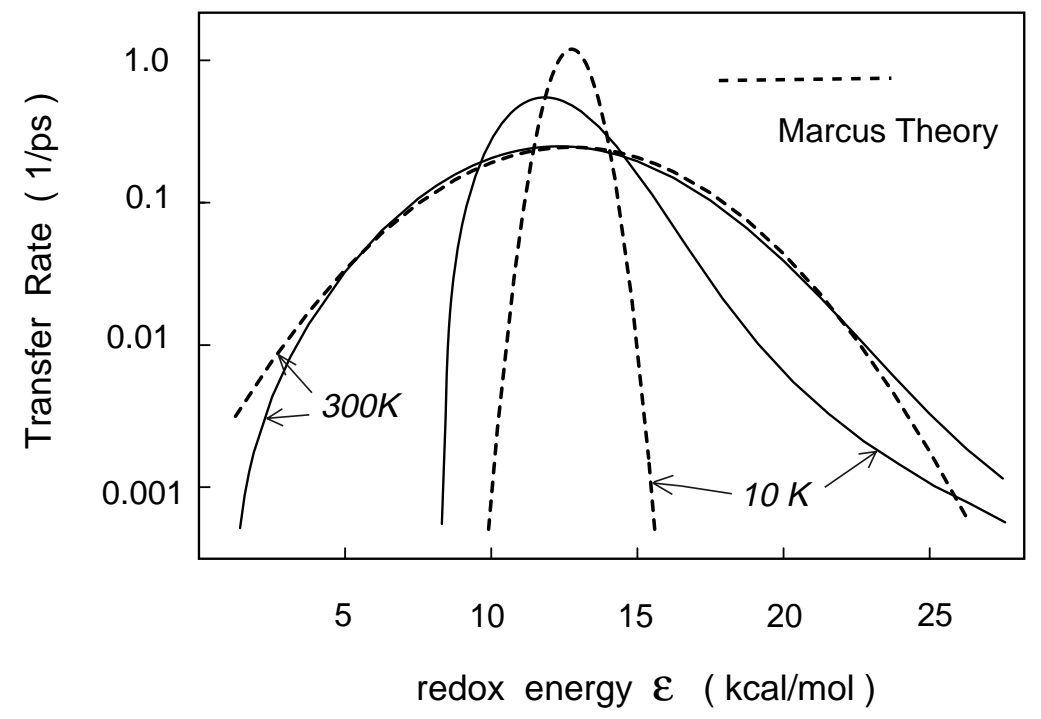

Figure 2: Comparison of electron transfer rates $k(\epsilon, T)$ shown as a function of $\epsilon$ evaluated in the framework of the spin-boson model (solid lines) and by Marcus theory (dashed lines) at temperatures $10 \mathrm{~K}$ and $300 \mathrm{~K}$. The functions are centered approximately around $\epsilon_{m}$. From [3].

35]. However, Fig. 3 demonstrates also that electron transfer at $E$-values slightly off the maximum position can yield a different temperature dependence than that of $k\left(E_{m}, T\right)$, namely temperature independence or a slight decrease of the rate with decreasing temperature. Such temperature dependence has also been observed for biological electron transfer [35]. As Nagarajan et al. reported in [35] the temperature dependence of the transfer rate resembles that of $k\left(E_{m}, T\right)$ in photosynthetic reaction centers of native bacteria and in (M)Y210F-mutants with tyrosine at the 210 position of the M-unit replaced by phenylalanine. However, a replacement of this tyrosine by isoleucine [(M)Y210I-mutant] yields a transfer rate which decreases like $k\left(E_{m}-\delta, T\right)$ shown in Fig. 3. This altered temperature dependence should be attributed to a shift of the redox potentials, i.e., $E_{m} \rightarrow E_{m}-\delta$.

The combination of simulation methods and analytical theory outlined in this lecture has proven to be a promising approach to describe redox processes in proteins. Neither approach by itself can be successful since, on the one hand, proteins are too heterogeneous and ill understood to be molded into simple models, on the other hand, simulation methods are blind, leaving one with too much information and as a result, with none. The present example, connecting a single simulated observable, the medium redox energy contribution $\Delta E(t)$, with a model of the quantum system, avoids superfluous or undetermined parameters and can be extended readily to other curve crossing processes in proteins. We like to mention, finally, that there have been numerous similar investigations of biological electron transfer [43, 44, 45, 46]. 


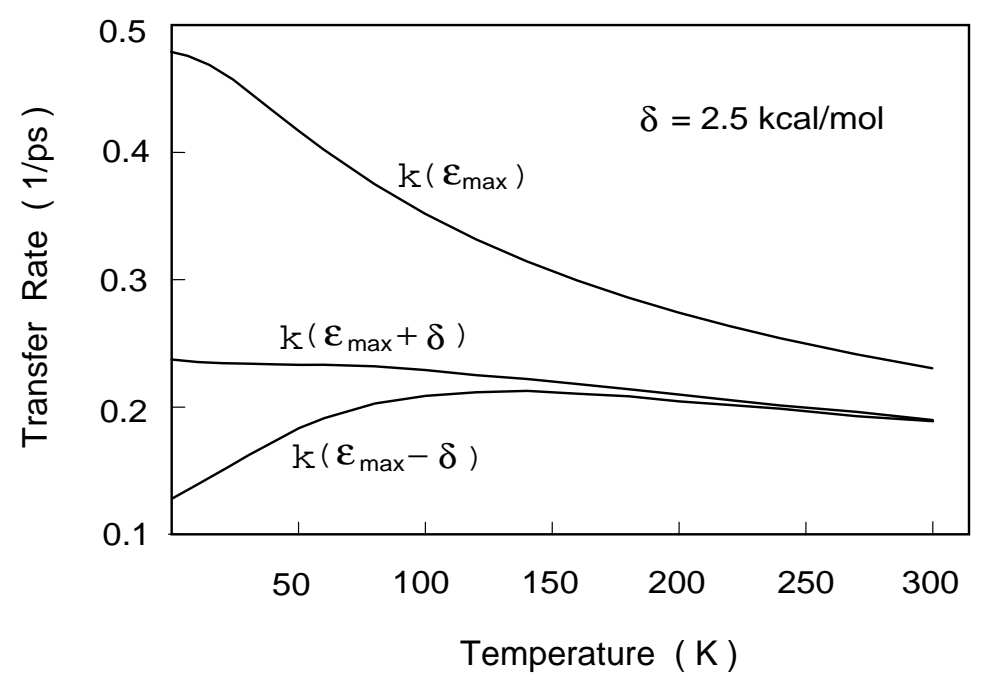

Figure 3: 1 Comparison of the temperature dependence of the maximum transfer rate of $k\left(\epsilon_{m}\right)$ and off-maximum value $k\left(\epsilon_{\max } \pm \delta\right)$, where $\delta=2.5 \mathrm{kcal} / \mathrm{mol} . k\left(\epsilon_{\max }, T\right)$ represents the fastest transfer rate of the system, the rates $k\left(\epsilon_{\max } \pm \delta, T\right)$ are slower since their $E$ values deviate from the optimal value $\epsilon_{\max }$. From [3].

\section{Acknowledgments}

I like to thank Dong $\mathrm{Xu}$ for a close collaboration on the subject of this lecture and Johannes Engelhardt for preparing lecture notes in 1985 which served us to write Sect. 5. We like to thank Robert Zwanzig for an unpublished manuscript with a derivation similar to ours in Sect. 6. This work was supported by grants from the National Institutes of Health (PHS 5 P41 RR05969-04) and the National Science Foundation (BIR-9318159 and ASC-8902829).

\section{A Numerical Evaluation of the Line Shape Func- tion}

We want to provide here an algorithm for the evaluation of the line shape function (46). The algorithm suggested is based on an expansion in terms of the right $[(u|m\rangle]$ and left $[\langle\tilde{n}| u)]$ eigenfunctions of the operator $\mathcal{L}=D \partial_{u} p_{0}(u) \partial_{u}\left[p_{0}(u)\right]^{-1}$ given in the Fokker-Planck Eq. (42). These eigenfunctions are in the present case

$$
\left(u|n\rangle=\frac{1}{\sqrt{\pi}} \frac{2^{-n}}{n !} e^{-u^{2}} H_{n}(u), \quad\langle\tilde{m}| u\right)=H_{m}(u)
$$

where $H_{m}$ denotes the Hermite polynomial. We assume in the following $\sigma=1$, i.e. measure $u$ in units of $\sigma$ and $D$ in units of $\sigma^{-2}$. In general, the eigenfucjtions and eigenvalues of the stochastic operator are not available in analytical form and 
an evaluation of the line shape function is based on discretization of the coordinate $u$ and of the operator $\mathcal{L}$. The eigenfunctions form a bi-orthogonal system [47], i.e., defining $\left.\langle\tilde{m} \mid n\rangle=i n t_{-\infty}^{+\infty} d u \angle m \mid u\right)(u|n\rangle$, the following property applies

$$
\langle\tilde{m} \mid n\rangle=\frac{1}{\sqrt{\pi}} \frac{2^{-n}}{n !} \int_{-\infty}^{+\infty} d u H_{m}(u) e^{-u^{2}} H_{n}(u)=\delta_{m n} .
$$

One can show that the function spaced spanned by the eigenfunctions is complete, i.e., any element of $\{f(u), u \in]-\infty, \infty[, f$ continuous $\}$ can be expanded. The eigenvalues of $\mathcal{L}$ follow from the property [47]

$$
\mathcal{L}|n\rangle=-2 m D|n\rangle,\langle\tilde{m}| \mathcal{L}^{\dagger}=-2 m D\langle\tilde{m}| .
$$

According to [47] holds

$$
\left(u|n+1\rangle=2 u\left(u|n\rangle-2 n\left(u|n-1\rangle,\langle\tilde{m}|u| n\rangle=\frac{1}{2} \delta_{m n+1}+n \delta_{m n-1} .\right.\right.\right.
$$

From this and Eq. 177 we obtain

$$
\left\langle\tilde{m}\left|i\left(u-u^{\prime}\right)-\mathcal{L}\right| n\right\rangle=\left(2 n D-i u^{\prime}\right) \delta_{m n}+\frac{i}{2} \delta_{m n+1}+i n \delta_{m n-1} \equiv B_{m n}\left(u^{\prime}\right)
$$

where $B$ is an infinite-dimensional, tri-diagonal matrix. Obviously

$$
\left(u|0\rangle=p_{o}(u),\langle\tilde{0}| u\right)=1
$$

Equation (46) yields then

$$
\mathcal{I}=\frac{1}{\pi} \operatorname{Re}\left[B^{-1}\left(u^{\prime}\right)\right]_{00} .
$$

In order to evaluate $\left[B^{-1}\left(u^{\prime}\right)\right]_{00}$ we start from

$$
\left(\begin{array}{ccc}
1 & 0 & \ldots
\end{array}\right) B^{-1}\left(u^{\prime}\right)\left(\begin{array}{c}
1 \\
0 \\
\vdots
\end{array}\right)=\left[B^{-1}\left(u^{\prime}\right)\right]_{00}
$$

which can be written as

$$
\left(\begin{array}{c}
1 \\
0 \\
\vdots
\end{array}\right)=B\left(u^{\prime}\right)\left(\begin{array}{c}
y_{0} \\
y_{1} \\
\vdots
\end{array}\right), y_{0}=\left[B^{-1}\left(u^{\prime}\right)\right]_{00} .
$$

The latter equation together with Eq. (179) can be written in matrix notation

$$
\left(\begin{array}{ccccccc}
-i u^{\prime} & \frac{i}{2} & 0 & \ldots & & \\
i & 2 D-i u^{\prime} & \frac{i}{2} & 0 & \ldots & \\
0 & 2 i & 4 D-i u^{\prime} & \frac{i}{2} & 0 & \ldots & \\
\vdots & \ddots & \ddots & \ddots & \ddots & \ddots & \ldots \\
0 & \ldots & 0 & 2 n i & 2 n D-i u^{\prime} & \frac{i}{2} & \ldots \\
\vdots & \vdots & \ldots & \ddots & \ddots & \ddots & \ddots
\end{array}\right)\left(\begin{array}{c}
y_{0} \\
y_{1} \\
y_{2} \\
\vdots \\
y_{n} \\
\vdots
\end{array}\right)=\left(\begin{array}{c}
1 \\
0 \\
0 \\
\vdots \\
0 \\
\vdots
\end{array}\right)
$$


For the numerical solution a finite dimension $N=2000$ is assumed for the matrix $B$.

The ensuing problem has been discussed in [48], albeit for a real matrix $B$. The use of a finite-dimensional matrix $B$ can only be justified for $u^{\prime} \ll N$.

\section{References}

[1] Klaus Schulten and Markus Tesch. Coupling of protein motion to electron transfer: Molecular dynamics and stochastic quantum mechanics study of photosynthetic reaction centers. Chem. Phys., 158:421-446, 1991.

[2] Dong Xu and Klaus Schulten. Coupling of protein motion to electron transfer in a photosynthetic reaction center: Investigating the low temperature behaviour in the framework of the spin-boson model. Chem. Phys., 182:91-117, 1994.

[3] Dong Xu and Klaus Schulten. Multi-mode coupling of protein motion to electron transfer in the photosynthetic reaction center: Spin-boson theory based on a classical molecular dynamics simulation. In J. Breton and A. Vermeglio, editors, The Photosynthetic Bacterial Reaction Center: II. Structure, Spectroscopy and Dynamics, pages 301-312. Plenum Press, New York, 1992.

[4] R. Kubo. Adv. Chem. Phys., 15:101, 1969.

[5] H. J. C. Berendsen and J. Mavri. Quantum simulation of reaction dynamics by density matrix evolution. J. Phys. Chem., 97:13464-13468, 1993.

[6] Treutlein, Klaus Schulten, J. Deisenhofer, H. Michel, Axel Brünger, and Martin Karplus. Molecular dynamics simulation of the primary processes in the photosynthetic reaction center of Rhodopseudomonas viridis. In J. Breton and A. Verméglio, editors, The Photosynthetic Bacterial Reaction Center: Structure and Dynamics, volume 149, pages 139-150. Plenum, New York, 1988.

[7] Herbert Treutlein, Klaus Schulten, Christoph Niedermeier, J. Deisenhofer, H. Michel, and D. Devault. Electrostatic control of electron transfer in the photosynthetic reaction center of Rhodopseudomonas viridis. In J. Breton and A. Verméglio, editors, The Photosynthetic Bacterial Reaction Center: Structure and Dynamics, volume 149, pages 369-377. Plenum, New York, 1988.

[8] R. A. Marcus. On the energy of oxidation-reduction reactions involving electron transfer. I. J. Chem. Phys., 24:966-978, 1956.

[9] R. A. Marcus. Electrostatic free energy and other properties of states having nonequilibrium polarization. II. J. Chem. Phys., 24:979-989, 1956. 
[10] Herbert Treutlein, Klaus Schulten, J.Deisenhofer, H.Michel, Axel Brünger, and Martin Karplus. Chromophore-protein interactions and the function of the photosynthetic reaction center: A molecular dynamics study. Proc. Natl. Acad. Sci. USA, 89:75-79, 1991.

[11] G.E. Uhlenbeck and L.S. Ohrnstein. Phys. Rev., 36:823, 1930. These papers are reprinted in Selected Papers on Noise and Stochastic Processes, ed. by N. Wax (Dover,New York 1954).

[12] S. Chandrasekhar. Rev. Mod. Phys., 15:1, 1943.

[13] Robert Bittl and Klaus Schulten. Length dependence of the magnetic field modulated triplet yield of photogenerated biradicals. Chem. Phys. Lett., 146:58-62, 1988.

[14] Marco Nonella and Klaus Schulten. Molecular dynamics simulation of electron transfer in proteins: Theory and application to $\mathrm{Q}_{A} \rightarrow \mathrm{Q}_{B}$ transfer in the photosynthetic reaction center. J. Phys. Chem., 95:2059-2067, 1991.

[15] Klaus Schulten and Robert Bittl. Probing the dynamics of a polymer with paramagnetic end groups by magnetic fields. J. Chem. Phys., 84:5155-5161, 1986.

[16] Robert Bittl and Klaus Schulten. A static ensemble approximation for stochastically modulated quantum systems. J. Chem. Phys., 90:1794-1803, 1989.

[17] Benno Pütz, Daniel Barsky, and Klaus Schulten. Edge enhancement by diffusion: Microscopic magnetic resonance imaging of an ultra-thin glass capillary. Chem. Phys. Lett., 183(5):391-396, 1991.

[18] Benno Pütz, Daniel Barsky, and Klaus Schulten. Edge enhancement by diffusion in microscopic magnetic resonance imaging. J. Magn. Res., 97:27-53, 1992.

[19] Walter Nadler and Klaus Schulten. Generalized moment expansion for Brownian relaxation processes. J. Chem. Phys., 82:151-160, 1985.

[20] Daniel Barsky, Benno Pütz, Klaus Schulten, J. Schoeniger, E. W. Hsu, and S. Blackband. Diffusional edge enhancement observed by NMR in thin glass capillaries. Chem. Phys. Lett., 200:88-96, 1992.

[21] J. J. Markham. Interaction of normal modes with electron traps. Rev. Mod. Phys., 31:956, 1959.

[22] J. Jortner. J. Chem. Phys., 64:4860-4867, 1976.

[23] D. DeVault. Quantum-mechanical tunneling in biological systems. Cambridge University press, Cambridge, UK, 1984. 
[24] A. O. Cardeira and A. J. Leggett. Quantum tunnelling in a dissipative system. J. Ann. Phys.(N.Y.), 149:374-456, 1983.

[25] A. O. Cardeira and A. J. Leggett. Path integral approach to quantum brownian motion. Physica A, 121:587-616, 1983.

[26] A.J. Leggett, S. Chakravarty, A.T. Dorsey, M.P.A. Fisher, A. Garg, and W. Zwerger. Dynamics of the dissipative two-state system. Rev. Mod. Phys., 59:2-86, 1987.

[27] Lord Rayleigh. The Theory of Sound, Vol. I, 2nd ed. MacMillan and Company Ltd., London, 1894.

[28] Lord Rayleigh. Scientific Papers, Vol. I, p491 and Vol. IV, p370. Cambridge University Press, Cambridge, England, 1899-1920.

[29] Ilya Rips and Joshua Jortner. Dynamic solvent effects on outer-sphere electron transfer. J. Chem. Phys., 87:2090-2104, 1987.

[30] J. S. Bader, R. A. Kuharski, and D. Chandler. Role of nuclear tunneling in aqueous ferrous-ferric electron transfer. J. Chem. Phys., 93:230-236, 1990.

[31] V. K. Thankappan. Quantum Mechanics. John Wiley \& Sons, New York, 1985.

[32] A. Garg, J. N. Onuchic, and V. Ambegaokar. Effect of friction on electron transfer in biomolecules. J. Chem. Phys., 83:4491-4503, 1985.

[33] J. N. Onuchic, D. N. Beratan, and J. J. Hopfield. Some aspects of electrontransfer reaction dynamics. J. Phys. Chem., 90:3707-3721, 1986.

[34] G. R. Fleming, J. L. Martin, and J. Breton. Rates of primary electron transfer in photosynthetic reaction centres and their mechanistic implications. Nature., 333:190, 1988.

[35] V. Nagarajan, W. W. Parson, D. Gaul, and C. Schenck. Effect of specific mutations of tyrosine- $(\mathrm{m}) 210$ on the primary photosynthetic electron-transfer process in rhodobacter sphaeroides. Proc. Natl. Acad. Sci. USA, 87:7888-7892, 1990.

[36] M. R. Gunner and P. Leslie Dutton. Temperature and $\Delta G_{o}$ dependence of the electron transfer from $B \mathrm{Ph}^{-}$to $Q_{a}$ in a reaction center protein from rhodobacter sphaeroides with different quinones as $Q_{a}$. J. Am. Chem. Soc., 111:3400-3412, 1989.

[37] R. A. Marcus and N. Sutin. Electron transfers in chemistry and biology. Biochem. Biophys. Acta, 811:265-322, 1985. 
[38] H. Sumi and R. A. Marcus. Dynamical effects in electron transfer reactions. J. Chem. Phys., 84:4894-4914, 1986.

[39] J. J. Hopfield. Electron transfer between biological molecules by thermally activated tunneling. Proc. Natl. Acad. Sci. USA, 71:3640-3644, 1974.

[40] M. Bixon and J. Jortner. Coupling of protein modes to electron transfer in bacterial photosynthesis. J. Phys. Chem., 90:3795-3800, 1986.

[41] J. L. Martin, J. Breton, J. C. Lambry, and G. Fleming. The primary electron transfer in photosynthetic purple bacteria: Long range electron transfer in the femtosecond domain at low temperature. In J. Breton and A. Vermeglio, editors, The Photosynthetic Bacterial Reaction Center: Structure and Dynamics, pages 195-203, New York and London, 1988. Plenum Press.

[42] C. Kirmaier and D. Holten. Temperature effects on the ground state absorption spectra and electron transfer kinetics of bacterial reaction centers. In J. Breton and A. Vermeglio, editors, The Photosynthetic Bacterial Reaction Center: Structure and Dynamics, pages 219-228, New York and London, 1988. Plenum Press.

[43] Arieh Warshel and Jenn-Kang Hwang. Simulations of the dynamics of electron transfer reactions in polar solvent: Semiclassical trajectories and dispersed polaron approaches. J. Chem. Phys., 84:4938-4957, 1986.

[44] A. Warshel, Z. T. Chu, and W. W. Parson. Dispersed polaron simulations of electron transfer in photosynthetic reaction center. Science, 246:112-116, 1989.

[45] Peter G. Wolynes. Dissipation, tunneling and adiabaticity criteria for curve crossing problems in the condenced phase. J. Chem. Phys., 86:1957-1966, 1987.

[46] C. Zheng, J. A. McCammon, and P. G. Wolynes. Proc. Natl. Acad. Sci. USA, 86:6441, 1989.

[47] M. Abramowitz and I.A. Stegun. Handbook of Mathematical Functions. Dover, New York, 1972.

[48] W.H. Press, B.P. Flannery, S.A. Teukolsky, and W.T. Vetterling. Numerical Recipes in C. Cambridge University Press, Melbourne, 1988. 